\title{
The Logarithmic Super Divergence and Statistical Inference : Asymptotic Properties
}

\author{
Avijit Maji, Abhik Ghosh and \\ Ayanendranath Basu
}

\begin{abstract}
Statistical inference based on divergence measures have a long history. Recently, Maji, Ghosh and Basu (2014) have introduced a general family of divergences called the logarithmic super divergence (LSD) family. This family acts as a superfamily for both of the logarithmic power divergence (LPD) family (eg. Renyi, 1961) and the logarithmic density power divergence (LDPD) family introduced by Jones et al. (2001). In this paper we describe the asymptotic properties of the inference procedures resulting from this divergence in discrete models. The properties are well supported by real data examples.
\end{abstract}

Key words: asymptotic properties, logarithmic density power divergence, logarithmic power divergence, logarithmic super divergence, $S$-divergence, statistical inference.

\section{Introduction}

The density-based minimum divergence approach has long been an important parametric inference tool. In this approach the closeness between the data and the model is measured by a density-based divergence between the data density and the model density, such as a $\chi^{2}$ type divergence or a $\phi$-divergence (Csiszár 1963, 1967a,b; Ali and Silvey 1966) or a Bregman divergence (Bregman, 1967). Apart from their natural appeal, most of these methods are very useful for their inherent robustness properties. A prominent member of the class of densitybased divergences is the Pearson's $\chi^{2}$ (Pearson, 1900) which started its journey from the very early days of formal research in statistics. From the robustness perspective, however, Beran's 1977 work is the first useful reference in the literature of density-based minimum divergence inference. In the present paper we focus on some variants of the power divergence (PD) measure of Cressie and

Indian Statistical Institute

203, B.T. Road, Kolkata-700108, India.

E-mail: avijit.maji@hotmail.com, abhianik@gmail.com, ayanendranath.basu@gmail.com. 
Read (1984) and the density power divergence (DPD) of Basu et al. (1998) and discuss various properties related to statistical inference based on a generalized superfamilies of these measures. The primary divergence class of logarithmic super divergences (LSDs) which is of interest to us in this paper has been proposed recently by Maji, Ghosh and Basu (2014). In the present paper we establish the theoretical asymptotic properties of the resulting statistical procedures.

The rest of the paper is organized as follows. Section 2 describes the logarithmic power divergence family and the logarithmic density power divergence family. Section 3 gives the form and the estimating equation of the logarithmic super divergence (LSD) family whereas Section 4 establishes the asymptotic distribution for LSD estimator. Section 5 gives some illustration of the proposed estimation procedure with real data. Section 6 gives the asymptotic distribution of the test statistic using LSD measure for both one sample and two sample problem and Section 7 provides a hypothesis testing example. Concluding remarks are in Section 8.

\section{The Logarithmic Power Divergence (LPD) and the Logarithmic Density Power Divergence (LDPD) Families}

Jones et al. (2001) described a class of divergence measures which do not require any nonparametric smoothing techniques for their construction. This family of divergences is given by

$$
\operatorname{LDPD}_{\beta}(g, f)=\log \int f^{1+\beta}-\left(1+\frac{1}{\beta}\right) \log \int f^{\beta} g+\frac{1}{\beta} \log \int g^{1+\beta},
$$

$\beta \geq 0$, where LDPD stands for logarithmic density power divergence. For the case $\beta=0$ we define

$$
\operatorname{LDPD}_{0}(g, f)=\lim _{\beta \rightarrow 0} \operatorname{LDPD}_{\beta}(g, f)=\lim _{\lambda \rightarrow 0} \operatorname{PD}_{\lambda}(g, f)=\operatorname{PD}_{0}(g, f),
$$

where the power divergence $(\mathrm{PD})$ measure has the form

$$
\mathrm{PD}_{\lambda}(g, f)=\frac{1}{\lambda(\lambda+1)} \int g\left[\left(\frac{g}{f}\right)^{\lambda}-1\right], \quad-\infty<\lambda<\infty .
$$

The family of divergences in (1) is similar to the density power divergence family given by

$$
\operatorname{DPD}_{\alpha}(g, f)=\int\left[f^{1+\alpha}-\left(1+\frac{1}{\alpha}\right) f^{\alpha} g+\frac{1}{\alpha} g^{1+\alpha}\right] \text { for } \alpha \geq 0 .
$$

The LDPD family may be recovered from the DPD by replacing the identity function with the logarithm function. In spite of the similarity between the forms of the DPD and the LDPD families, Jones et al. (2001) had originally 
developed the latter as a special case of an estimation method proposed by Windham (1995). Following the connection shown between PD and DPD by Patra et al. (2013), Maji, Chakraborty and Basu (2014) have recently shown that the same sort of connection exists between LDPD and the logarithmic power divergence (LPD) family. To show this connection, the LDPD measure can be written as

$$
\begin{aligned}
\operatorname{LDPD}_{\beta}(g, f)= & \log \int f^{1+\beta}-\left(1+\frac{1}{\beta}\right) \log \int\left(\frac{g}{f}\right) f^{1+\beta} \\
& +\frac{1}{\beta} \log \int\left(\frac{g}{f}\right)^{1+\beta} f^{1+\beta} .
\end{aligned}
$$

Replacing the $f^{1+\beta}$ term with $f$ in each of the three terms on the right hand side of (4) leads to the density-based divergence $\frac{1}{\beta} \log \int \frac{g^{1+\beta}}{f^{\beta}}$. After standardizing this further we express this divergence as $\frac{1}{\beta(\beta+1)} \log \int \frac{g^{1+\beta}}{f^{\beta}}$. This family of divergences will be called the logarithmic power divergence family by Maji, Chakraborty and Basu (2014). Using a different symbol for the tuning parameter, this family has the form

$$
\operatorname{LPD}_{\gamma}(g, f)=\frac{1}{\gamma(\gamma+1)} \log \int \frac{g^{1+\gamma}}{f^{\gamma}}, \gamma \in \mathbb{R} .
$$

The limiting forms as $\gamma \rightarrow 0$ and $\gamma \rightarrow-1$ generate, respectively, the likelihood disparity $\mathrm{LD}$ (or $\mathrm{PD}_{0}$ ) and the Kullback-Leibler divergence KLD (or $\mathrm{PD}_{-1}$ ). These divergences have the form

$$
\begin{aligned}
\mathrm{LD}(g, f) & =\int g \log \left(\frac{g}{f}\right), \\
\operatorname{KLD}(g, f) & =\int f \log \left(\frac{f}{g}\right) .
\end{aligned}
$$

For any other value of $\gamma$ the LPD measure can be seen to be a function of the PD measure at the same value of $\gamma$. Specifically,

$$
\operatorname{LPD}_{\gamma}(g, f)=\frac{1}{\gamma(\gamma+1)} \log \left[\gamma(\gamma+1) \mathrm{PD}_{\gamma}(g, f)+1\right]
$$

Apart from being briefly considered by Renyi as a measure of the amount of information (Renyi, 1961), the LPD family is a member of the $(h, \phi)$ divergence family (eg. Pardo, 2006), where $h(x)=\frac{1}{r(r+1)}[r(r+1) \log x+1]$.

\section{The Logarithmic Super Divergence}

We now define the Logarithmic Super Divergence (or Logarithmic $S$-Divergence) introduced in Maji, Ghosh and Basu (2014) and establish the 
asymptotic properties of the procedures resulting from it. The Logarithmic $S$-Divergence (LSD) is defined (Maji, Ghosh and Basu, 2014) as

$$
\begin{aligned}
\operatorname{LSD}_{\beta, \gamma}(g, f)= & \frac{1}{A} \log \int f^{1+\beta}-\frac{1+\beta}{A B} \log \int f^{B} g^{A} \\
& +\frac{1}{B} \log \int g^{1+\beta}, \quad \beta>0,-\infty<\gamma<\infty,
\end{aligned}
$$

where

$$
\begin{aligned}
A & =1+\gamma(1-\beta) \\
\text { and } B & =\beta-\gamma(1-\beta) .
\end{aligned}
$$

For $\beta=0(A=1+\gamma, B=-\gamma)$, this family coincides with the logarithmic power divergence family of (5) with parameter $\gamma$, while $\gamma=0$ gives the logarithmic density power divergence family in (1) with parameter $\beta$.

Replacing the logarithmic function with the identity function in (9) generates the divergence

$$
S_{\alpha, \lambda}(g, f)=\frac{1}{A} \int f^{1+\alpha}-\frac{1+\alpha}{A B} \int f^{B} g^{A}+\frac{1}{B} \int g^{1+\alpha},
$$

with $\beta$ and $\gamma$ being replaced by $\alpha$ and $\lambda$ respectively. This divergence is known as the $S$-divergence and has been introduced by Ghosh et al. (2013).

\subsection{Estimating Equation}

Consider a parametric class of model densities $\left\{f_{\theta}: \theta \in \Theta \subseteq \mathbb{R}^{p}\right\}$ and suppose that our interest is in estimating $\theta$. Let $G$ denote the distribution function corresponding to the true density $g$. The minimum LSD functional $T_{\beta, \gamma}(G)$ at $G$ is defined as

$$
\operatorname{LSD}_{\beta, \gamma}\left(g, f_{T_{\beta, \gamma}(G)}\right)=\min _{\theta \in \Theta} \operatorname{LSD}_{\beta, \gamma}\left(g, f_{\theta}\right) .
$$

It takes the value $\theta$ when the true density $g=f_{\theta}$ is in the model; when it does not, $\theta_{\beta, \gamma}^{g}=T_{\beta, \gamma}(G)$ represents the best fitting parameter, and $f_{\theta g}$ is the model element closest to $g$ in terms of logarithmic super divergence. For simplicity of notation, we suppress the subscript $\beta, \gamma$ in $\theta_{\beta, \gamma}^{g}$. A simple differentiation gives us the estimating equation for $\theta$, which is

$$
\frac{\int f_{\theta}^{1+\beta} u_{\theta}}{\int f_{\theta}^{1+\beta}}=\frac{\int f_{\theta}^{B} g^{A} u_{\theta}}{\int f_{\theta}^{B} g^{A}}
$$

For $\beta=0(A=1+\gamma, B=-\gamma)$, the equation thus becomes the same as the estimating equation of the logarithmic power divergence family with parameter $\gamma$. For $\gamma=0(A=1, B=\beta)$, on the other hand, it is the estimating equation for the LDPD measure. Here $u_{\theta}(x)=\frac{\partial}{\partial \theta} \log f_{\theta}(x)$ is the likelihood score function. 


\section{Asymptotic Properties of the Minimum LSD Estimators in} Discrete Models

Under the parametric set-up of Section 3.1, consider a discrete family of distributions. We will use the term "density function" generally for the sake of a unified notation, irrespective of whether the distribution is discrete or continuous. Let $X_{1}, \ldots, X_{n}$ be a random sample from the true distribution having density function $g$. Representing the logarithmic $S$-divergence in terms of the parameter $\beta$ and $\gamma$ (as given in Section 3), let $\hat{\theta}_{\beta, \gamma}$ be the estimator obtained by minimizing $\operatorname{LSD}_{\beta, \gamma}\left(\hat{g}, f_{\theta}\right)$ over $\theta \in \Theta$, where $\hat{g}$ is a suitable nonparametric density estimate of $g$; in the discrete case the vector of relative frequencies based on the sample data is the canonical choice for $\hat{g}$. For similar theoretical properties of the minimum $S$-divergence estimator, see Ghosh (2014).

\subsection{Asymptotic Properties}

Suppose $X_{1}, X_{2}, \ldots, X_{n}$ are $n$ independent and identically distributed observations from a discrete distribution $G$ modeled by $\mathcal{F}=\left\{F_{\theta}: \theta \in \Theta \subseteq \mathbb{R}^{p}\right\}$ and let the distribution have support $\mathcal{X}=\{0,1,2, \ldots\}$ without loss of generality. Denote the relative frequency at $x$ as obtained from the data by $r_{n}(x)=\frac{1}{n} \sum_{i=1}^{n} \chi\left(X_{i}=x\right)$ where $\chi(A)$ is the indicator of $A$. The minimum LSD estimator is then obtained by minimizing the LSD measure between the data density $r_{n}$ and the model density $f_{\theta}$ with respect to $\theta$.

Minimizing $\operatorname{LSD}_{\beta, \gamma}\left(r_{n}, f_{\theta}\right)$ with respect to $\theta$ is equivalent to minimizing $H_{n}(\theta)$ where

$$
H_{n}(\theta)=\frac{1}{1+\beta}\left[\frac{1}{A} \log \sum_{x} f_{\theta}^{1+\beta}(x)-\frac{1+\beta}{A B} \log \sum_{x} f_{\theta}^{B}(x) r_{n}^{A}(x)\right] .
$$

Now,

$$
\nabla H_{n}(\theta)=\frac{1}{A}\left[\frac{\sum_{x} f_{\theta}^{1+\beta}(x) u_{\theta}(x)}{\sum_{x} f_{\theta}^{1+\beta}(x)}-\frac{\sum_{x} f_{\theta}^{B}(x) r_{n}^{A}(x) u_{\theta}(x)}{\sum_{x} f_{\theta}^{B}(x) r_{n}^{A}(x)}\right],
$$

where $\nabla$ is the gradient with respect to $\theta$. Equating the above to zero, the estimating equation becomes

$$
\sum_{x} K\left(\delta_{n}(x)\right) f_{\theta}^{1+\beta}(x) w_{\theta}(x)=0
$$

where $\delta_{n}(x)=\frac{r_{n}(x)}{f_{\theta}(x)}, \quad \delta_{g}(x)=\frac{g(x)}{f_{\theta}(x)}, \quad w_{\theta}(x)=\left[B(\theta) u_{\theta}(x)-A(\theta)\right], \quad K(\delta)=$ $\delta^{A}-1, \quad A(\theta)=\sum_{x} f_{\theta}^{1+\beta}(x) u_{\theta}(x)$ and $B(\theta)=\sum_{x} f_{\theta}^{1+\beta}(x)$. 
Define

$$
\begin{aligned}
J_{g}= & E_{g}\left[w_{\theta^{g}}(X) u_{\theta^{g}}^{T}(X) K^{\prime}\left(\delta_{g}^{g}(X)\right) f_{\theta^{g}}^{\beta}(X)\right] \\
& -\sum_{x} K\left(\delta_{g}^{g}(x)\right) f_{\theta}^{1+\beta}(x) \nabla w_{\theta^{g}}(x) \\
& -(1+\beta) \sum_{x} K\left(\delta_{g}^{g}(X)\right) f_{\theta}^{1+\beta}(x) w_{\theta^{g}}(x) u_{\theta^{g}}(x) . \\
V_{g}= & V_{g}\left[K^{\prime}\left(\delta_{g}^{g}(X)\right) f_{\theta^{g}}^{\beta}(X) w_{\theta^{g}}(X)\right] .
\end{aligned}
$$

We now present the necessary assumptions for our asymptotic results.

1. The model family $F_{\theta}$ is identifiable which means that different values of the parameter must generate different probability distributions of the observable variables.

2. The probability density function $f_{\theta}$ of the model distribution have common support so that the set $\mathcal{X}=\left\{x: f_{\theta}(x)>0\right\}$ is independent of $\theta$. Also the true distribution $g$ is compatible with the model family.

3. There exists an open subset $\omega \subset \Theta$ for which the best fitting parameter $\theta^{g}$ is an interior point and for almost all $x$, the density $f_{\theta}(x)$ admits all third derivatives of the type $\nabla_{j k l} f_{\theta}(x)$ for all $\theta \in \omega$. Here the subscripts $j, k, l$ of $\nabla$ represent the indicated partial derivatives.

4. The matrix $\frac{1+\beta}{A} J_{g}$ is positive definite.

5. The quantities $\sum_{x} g^{1 / 2}(x) f_{\theta}^{\beta}(x)\left|u_{j \theta}(x)\right|, \sum_{x} g^{1 / 2}(x) f_{\theta}^{\beta}(x)\left|w_{j \theta}(x)\right|$, $\sum_{x} g^{1 / 2}(x) f_{\theta}^{\beta}(x)\left|u_{j \theta}(x)\right|\left|u_{k \theta}(x)\right|$ and $\sum_{x} g^{1 / 2}(x) f_{\theta}^{\beta}(x)\left|u_{j k \theta}(x)\right|$ are bounded for all $j, k$ and for all $\theta \in \omega$.

6 . For almost all $x$, there exists functions $M_{j k l}(x), M_{j k, l}(x), M_{j, k, l}(x)$, possibly depending on $\beta$ that dominate, in absolute value, $f_{\theta}^{\beta}(x) u_{j k l \theta}(x)$, $f_{\theta}^{\beta}(x) u_{j k \theta}(x) u_{l \theta}(x)$ and $f_{\theta}^{\beta}(x) u_{j \theta}(x) u_{k \theta}(x) u_{l \theta}(x)$ respectively for all $j, k, l$ and that are uniformly bounded in expectation with respect to $g$ and $f_{\theta}$ for all $\theta \in \omega$, where the subscripts of $u$ denote the indicated partial derivatives of the score function.

7. The function $\left(\frac{g(x)}{f_{\theta}(x)}\right)^{A-1}$ is uniformly bounded (by, say, $C$ ) for all $\theta \in \omega$.

To prove the consistency and asymptotic normality of the minimum LSD estimator, we will assume, for the rest of the paper, that the seven conditions stated above are satisfied.

Based on the above assumptions we now start the proofs of the required results. The proofs are primarily along the lines of Lindsay (1994) and Basu et al. (2011). Define $\eta_{n}(x)=\sqrt{n}\left(\sqrt{\delta}_{n}-\sqrt{\delta}_{g}\right)^{2}$. We then have the following lemmas.

Lemma 1. For any $k \in[0,2]$, we have

$$
\text { 1. } E\left[\eta_{n}^{k}(x)\right] \leq n^{\frac{k}{2}} E\left[\left|\delta_{n}(X)-\delta_{g}(X)\right|\right]^{k} \leq\left[\frac{g(x)(1-g(x))}{f_{\theta}^{2}(x)}\right]^{\frac{k}{2}} \text {. }
$$


2. $E\left[\left|\delta_{n}(X)-\delta_{g}(X)\right|\right] \leq \frac{2 g(x)(1-g(x))}{f_{\theta}(x)}$.

Proof. For $a, b \geq 0$, we have the inequality $(\sqrt{a}-\sqrt{b})^{2} \leq|a-b|$. So we get

$$
\begin{aligned}
E\left[\eta_{n}^{k}(x)\right] & =n^{\frac{k}{2}} E\left[\left(\sqrt{\delta}_{n}-\sqrt{\delta}_{g}\right)^{2}\right]^{k} \\
& \leq n^{\frac{k}{2}} E\left[\left|\delta_{n}-\delta_{g}\right|\right]^{k} .
\end{aligned}
$$

For the next part see that, $n r_{n}(x) \sim \operatorname{Bin}(n, g(x))$ for all $x$. Now, for any $k \in[0,2]$, we get by the Lyapunov's inequality that

$$
\begin{aligned}
E\left[\left|\delta_{n}(X)-\delta_{g}(X)\right|\right]^{k} & \leq\left[E\left(\delta_{n}(X)-\delta_{g}(X)\right)^{2}\right]^{\frac{k}{2}} \\
& =\frac{1}{f_{\theta}^{k}(x)}\left[E\left(r_{n}(X)-g(X)\right)^{2}\right]^{\frac{k}{2}} \\
& =\frac{1}{f_{\theta}^{k}(x)}\left[\frac{g(x)(1-g(x))}{n}\right]^{\frac{k}{2}} .
\end{aligned}
$$

For the second part, note that

$$
\begin{aligned}
E\left[\left|\delta_{n}(X)-\delta_{g}(X)\right|\right] & =\frac{1}{f_{\theta}^{k}(x)}\left[E\left|r_{n}(X)-g(X)\right|\right]^{\frac{k}{2}} \\
& \leq \frac{2 g(x)(1-g(x))}{f_{\theta}(x)}
\end{aligned}
$$

where the last inequality follows from the result about the mean-deviation of a Binomial random variable.

Lemma 2. $E\left[\eta_{n}^{k}(x)\right] \rightarrow 0$, as $n \rightarrow \infty$, for $k \in[0,2)$.

Proof. This follows from Theorem 4.5.2 of Chung (1974) by noting that $n^{1 / 4}\left(r_{n}^{1 / 2}(x)-g^{1 / 2}(x)\right) \rightarrow 0$ with probability one for each $x \in \mathcal{X}$ and by the Lemma 1(1), $\sup _{n} E\left[\eta_{n}^{k}(x)\right]$ is bounded.

Let us now define, $a_{n}(x)=K\left(\delta_{n}(x)\right)-K\left(\delta_{g}(x)\right)$ and $b_{n}(x)=\left(\delta_{n}(x)-\right.$ $\left.\delta_{g}(x)\right) K^{\prime}\left(\delta_{g}(x)\right)$.

We will need the limiting distributions of

$$
S_{1 n}=\sqrt{n} \sum_{x} a_{n}(x) f_{\theta}^{1+\beta}(x) w_{\theta}(x)
$$

and

$$
S_{2 n}=\sqrt{n} \sum_{x} b_{n}(x) f_{\theta}^{1+\beta}(x) w_{\theta}(x) .
$$

Define $\tau_{n}(x)=\sqrt{n}\left|a_{n}(x)-b_{n}(x)\right|$.

Lemma 3. Suppose Assumption 5 holds. Then $E\left|S_{1 n}-S_{2 n}\right| \rightarrow 0$ as $n \rightarrow \infty$, and hence $S_{1 n}-S_{2 n} \stackrel{P}{\rightarrow} 0$ as $n \rightarrow \infty$. 
Proof. By Lemma 2.15 of Basu et al. (2011) [or, Lindsay(1994), Lemma 25], there exists some positive constant $\beta$ such that

$$
\tau_{n}(x) \leq \beta \sqrt{n}\left(\sqrt{\delta}_{n}-\sqrt{\delta}_{g}\right)^{2}=\beta \eta_{n}(x) .
$$

Also, by Lemma $1, E\left[\tau_{n}(x)\right] \leq \beta \frac{g^{1 / 2}(x)}{f_{\theta}(x)}$.

And by Lemma 2, $E\left[\tau_{n}(x)\right]=\beta E\left[\eta_{n}(x)\right] \rightarrow 0$ as $n \rightarrow \infty$. Thus we get,

$$
\begin{aligned}
E\left|S_{1 n}-S_{2 n}\right| & \leq \sum_{x} E\left[\tau_{n}(x)\right] f_{\theta}^{1+\beta}(x)\left|w_{\theta}(x)\right| \\
& \leq \beta \sum_{x} g^{1 / 2}(x) f_{\theta}^{\beta}(x)\left|w_{\theta}(x)\right| \\
& <\infty \quad \text { (by Assumption 5). }
\end{aligned}
$$

So, by the Dominated Convergence Theorem (DCT), $E\left|S_{1 n}-S_{2 n}\right| \rightarrow 0$ as $n \rightarrow \infty$. Hence, by Markov's inequality, $S_{1 n}-S_{2 n} \stackrel{P}{\rightarrow} 0$ as $n \rightarrow \infty$.

Lemma 4. Suppose $V_{g}=V_{g}\left[K^{\prime}\left(\delta_{g}(X)\right) f_{\theta}^{\beta}(X) w_{\theta}(X)\right]$ is finite. Then

$$
S_{1 n} \rightarrow N\left(0, V_{g}\right) \text {. }
$$

Proof. By Lemma 3, the asymptotic distribution of $S_{1 n}$ and $S_{2 n}$ are the same. Now, we have

$$
\begin{aligned}
S_{2 n} & =\sqrt{n} \sum_{x}\left(\delta_{n}(x)-\delta_{g}(x)\right) K^{\prime}\left(\delta_{g}(x)\right) f_{\theta}^{1+\beta}(x) w_{\theta}(x) \\
& =\sqrt{n} \sum_{x}\left(r_{n}(x)-g(x)\right) K^{\prime}\left(\delta_{g}(x)\right) f_{\theta}^{\beta}(x) w_{\theta}(x) \\
& =\sqrt{n}\left(\frac{1}{n} \sum_{i=1}^{n}\left[K^{\prime}\left(\delta_{g}\left(X_{i}\right)\right) f_{\theta}^{\beta}\left(X_{i}\right) w_{\theta}\left(X_{i}\right)-E_{g}\left\{K^{\prime}\left(\delta_{g}(X)\right) f_{\theta}^{\beta}(X) w_{\theta}(X)\right\}\right]\right) \\
& \rightarrow N\left(0, V_{g}\right) \quad(\text { by the Central Limit Theorem). }
\end{aligned}
$$

We will now consider the final theorem of this section about the consistency and asymptotic normality of the minimum LSD estimator.

Theorem 5. Under Assumptions $1-7$, there exists a consistent sequence $\theta_{n}$ of roots to the minimum LSD estimating equation (12). Also, the asymptotic distribution of $\sqrt{n}\left(\theta_{n}-\theta_{g}\right)$ is $p$-dimensional normal with mean 0 and variance $J_{g}^{-1} V_{g} J_{g}^{-1}$.

Proof. Because of the lengthy and somewhat messy calculations, the proof of consistency has been put in Appendix. 
Proof of the asymptotic Normality : For the Asymptotic normality, we expand

$$
\sum_{x} K\left(\delta_{n}(x)\right) f_{\theta}^{1+\beta}(x) w_{\theta}(x)
$$

in Taylor series about $\theta=\theta^{g}$ to get

$$
\begin{aligned}
& \sum_{x} K\left(\delta_{n}(x)\right) f_{\theta}^{1+\beta}(x) w_{\theta}(x) \\
= & \sum_{x} K\left(\delta_{n}^{g}(x)\right) f_{\theta^{g}}^{1+\beta}(x) w_{\theta^{g}}(x)+\left.\sum_{k}\left(\theta_{k}-\theta_{k}^{g}\right) \nabla_{k}\left(\sum_{x} K\left(\delta_{n}(x)\right) f_{\theta}^{1+\beta}(x) w_{\theta}(x)\right)\right|_{\theta=\theta^{g}} \\
& +\left.\frac{1}{2} \sum_{k, l}\left(\theta_{k}-\theta_{k}^{g}\right)\left(\theta_{l}-\theta_{l}^{g}\right) \nabla_{k l}\left(\sum_{x} K\left(\delta_{n}(x)\right) f_{\theta}^{1+\beta}(x) w_{\theta}(x)\right)\right|_{\theta=\theta^{\prime}}
\end{aligned}
$$

where, $\theta^{\prime}$ lies in between $\theta$ and $\theta^{g}$. Now, let $\theta_{n}$ be the solution of the minimum LSD estimating equation, which can be assumed to be consistent. Replace $\theta$ by $\theta_{n}$ in above (18) so that the LHS of the equation becomes zero and hence we get

$$
\begin{gathered}
-\sqrt{n} \sum_{x} K\left(\delta_{n}^{g}(x)\right) f_{\theta^{g}}^{1+\beta}(x) w_{\theta^{g}}(x)=\sqrt{n} \sum_{k}\left(\theta_{n k}-\theta_{k}^{g}\right) \times \\
\left\{\left.\nabla_{k}\left(\sum_{x} K\left(\delta_{n}(x)\right) f_{\theta}^{1+\beta}(x) w_{\theta}(x)\right)\right|_{\theta=\theta^{g}}+\right. \\
\left.\left.\frac{1}{2} \sum_{l}\left(\theta_{n l}-\theta_{l}^{g}\right) \nabla_{k l}\left(\sum_{x} K\left(\delta_{n}(x)\right) f_{\theta}^{1+\beta}(x) w_{\theta}(x)\right)\right|_{\theta=\theta^{\prime}}\right\} .
\end{gathered}
$$

Note that, the first term within the bracketed quantity in the RHS of above (19) converges to $J_{g}$ with probability tending to one, while the second bracketed term is an $o_{p}(1)$ term (as proved in the proof of consistency part). Also, by using the Lemma 4, we get that

$$
\begin{aligned}
& \sqrt{n} \sum_{x} K\left(\delta_{n}^{g}(x)\right) f_{\theta^{g}}^{1+\beta}(x) w_{\theta^{g}}(x) \\
= & \sqrt{n} \sum_{x}\left[K\left(\delta_{n}^{g}(x)\right)-K\left(\delta_{g}^{g}(x)\right)\right] f_{\theta^{g}}^{1+\beta}(x) w_{\theta^{g}}(x) \text { (Using estimating equation) } \\
= & \left.S_{1 n}\right|_{\theta=\theta^{g}} \rightarrow N_{p}\left(0, V_{g}\right) .
\end{aligned}
$$

Therefore, by Lehmann(1983, Lemma 4.1), $\sqrt{n}\left(\theta_{n}-\theta^{g}\right)$ has asymptotic distribution as $N_{p}\left(0, J_{g}^{-1} V_{g} J_{g}^{-1}\right)$. 
A very interesting observation that follows from the asymptotic distribution just established is that the asymptotic distribution of the estimator is independent of the parameter $\gamma$. Yet the behavior of the estimator varies widely with $\gamma$ under the presence of outliers. Here we briefly report the findings reported by Maji, Ghosh and Basu (2014) in this connection, which is at least partially indicated by the results of the current and the subsequent section. Maji, Ghosh and Basu (2014) have observed that the first order influence function of the minimum LSD estimator is independent of $\gamma$, predicting that the robustness properties of the minimum LSD estimators are similar for each value of $\gamma$. This is immediately contradicted by the other results of Maji, Ghosh and Basu (2014) as well as the next section of the current article. Further exploration by Maji, Ghosh and Basu (2014) indicate that the second order influence function gives a much more accurate picture of the robustness of these estimators. This clearly indicates the limitation of the first order influence function in quantifying the robustness of the estimators in this case. In fact the second order influence analysis (see Maji, Ghosh and Basu, 2014 for details) shows that the limitation of the first order influence function can go both ways - it can fail to indicate the stability of a robust estimator, and can also describe a highly unstable estimator as a robust one.

\section{Examples}

\subsection{An Example with a Geometric Model}

The data set given in Basu et al. (2011), Table 2.4, represent the cases of peritonitis for 390 kidney patients. Following Basu et al. (2011) we use a geometric model with parameter $\theta$ (success probability) as our target distribution. A quick look at the data reveals that a geometric model with $\theta=0.5$ may fit the data well. We fit a geometric model with parameter $\theta$ using the LSD measure for various combinations of $\beta$ and $\gamma$. We can treat the two observations at 10 and 12 as mild to moderate outliers. We have evaluated the minimum LSD estimator in this case for the full data as well as the outlier deleted data. The estimates are presented shown in Tables 1 and 2 respectively. The estimates highlight an interesting point; for $\gamma<0$ or for larger values of $\beta$ with $\gamma \geq 0$ the parameter estimates are close for full data and outlier deleted data. However for $\gamma>0$ and $\beta$ small, the presence or absence of the outliers do not lead to a substantially larger difference. This gives a clear indication about which combinations of the $(\beta, \gamma)$ values keep the estimators stable and which are the ones that are easily affected.

\subsection{An Example with a Poisson Model}

This example gives us the observed frequencies and corresponding estimated frequencies (Table 3) for several minimum LSD estimators under the Poisson 
Table 1 The estimates of the parameter of the geometric model for different values of $\gamma$ and $\beta$ for the Peritonitis Incidence Data with outlier

\begin{tabular}{ccccccc}
\hline$\gamma \downarrow \beta \rightarrow$ & 0 & 0.2 & 0.4 & 0.6 & 0.8 & 1 \\
\hline-1 & - & 0.518 & 0.511 & 0.511 & 0.513 & 0.515 \\
-0.7 & 0.519 & 0.51 & 0.509 & 0.51 & 0.512 & 0.515 \\
-0.5 & 0.510 & 0.508 & 0.508 & 0.51 & 0.512 & 0.515 \\
-0.3 & 0.504 & 0.505 & 0.507 & 0.51 & 0.512 & 0.515 \\
-0.1 & 0.499 & 0.503 & 0.506 & 0.509 & 0.512 & 0.515 \\
0 & 0.496 & 0.502 & 0.506 & 0.509 & 0.512 & 0.515 \\
0.5 & 0.48 & 0.496 & 0.504 & 0.508 & 0.512 & 0.515 \\
1 & 0.461 & 0.486 & 0.501 & 0.507 & 0.512 & 0.515 \\
1.3 & 0.45 & 0.479 & 0.499 & 0.507 & 0.511 & 0.515 \\
1.7 & 0.438 & 0.469 & 0.495 & 0.506 & 0.511 & 0.515 \\
2 & 0.43 & 0.461 & 0.491 & 0.505 & 0.511 & 0.515 \\
\hline
\end{tabular}

Table 2 The estimates of the parameter of the geometric model for different values of $\gamma$ and $\beta$ for the Peritonitis Incidence Data without the two outliers

\begin{tabular}{ccccccc}
\hline$\gamma \downarrow \beta \rightarrow$ & 0 & 0.2 & 0.4 & 0.6 & 0.8 & 1 \\
\hline-1 & - & 0.521 & 0.512 & 0.511 & 0.513 & 0.515 \\
-0.7 & 0.526 & 0.513 & 0.51 & 0.511 & 0.513 & 0.515 \\
-0.5 & 0.518 & 0.511 & 0.509 & 0.51 & 0.512 & 0.515 \\
-0.3 & 0.513 & 0.509 & 0.508 & 0.51 & 0.512 & 0.515 \\
-0.1 & 0.510 & 0.508 & 0.508 & 0.51 & 0.512 & 0.515 \\
0 & 0.509 & 0.507 & 0.508 & 0.51 & 0.512 & 0.515 \\
0.5 & 0.505 & 0.505 & 0.506 & 0.509 & 0.512 & 0.515 \\
1 & 0.501 & 0.503 & 0.505 & 0.508 & 0.512 & 0.515 \\
1.3 & 0.5 & 0.501 & 0.504 & 0.508 & 0.512 & 0.515 \\
1.7 & 0.498 & 0.5 & 0.504 & 0.508 & 0.511 & 0.515 \\
2 & 0.496 & 0.499 & 0.503 & 0.507 & 0.511 & 0.515 \\
\hline & & & & & &
\end{tabular}

model for a sex linked recessive lethal test in drosophila (fruit flies) exposed to a certain chemical. For each of several male flies one samples about 100 daughter flies, and then determines the frequency of the number of daughter flies having a recessive lethal mutation in its $X$-chromosome. The data represent a frequency of frequencies; refer to Woodruff et al. (1984) for details. There is a possible case of outliers corresponding to the observations at $x=3,4$.

Table 3 provides the estimators and the predicted frequencies for a small number of $\gamma, \beta$ combinations, together with the fits of the maximum likelihood estimator (denoted by ML) and the outlier deleted maximum likelihood estimator obtained by removing the two outliers (denoted by ML+D). Clearly the estimators (and the estimated frequencies) are substantially different for the ML and ML+D cases, demonstrating that the maximum likelihood estimator is significantly affected by the presence of these outliers. Also apart from the ML, the $(\gamma=1, \beta=0.1)$ combination leads to highly unstable estimators. While Table 3 provides a small number of $(\gamma, \beta)$ combinations, a large selection is presented in Table 4, where the salient features may be described as follows: 
Table 3 Fits of the Poisson model to the Drosophila Data using several estimation methods

\begin{tabular}{lcccccccc}
\hline & \multicolumn{7}{c}{ Recessive lethal count } \\
\cline { 2 - 8 } & 0 & 1 & 2 & 3 & 4 & $\geq 5$ & $\theta$ \\
Observed & 23 & 3 & 0 & 1 & 1 & 0 & \\
ML & 19.59 & 7.00 & 1.25 & 0.15 & 0.01 & - & 0.3571 \\
ML+D & 24.95 & 2.88 & 0.17 & 0.01 & - & - & 0.1154 \\
$\operatorname{LSD}_{\gamma=1} \& \quad \beta=0.1$ & 14.90 & 9.40 & 2.97 & 0.62 & 0.10 & 0.01 & 0.6311 \\
$\operatorname{LSD}_{\gamma=-1} \& \quad \beta=0.1$ & 25.95 & 1.98 & 0.07 & - & - & - & 0.0762 \\
$\operatorname{LSD}_{\gamma=1} \& \quad \beta=1$ & 24.59 & 3.19 & 0.21 & 0.01 & - & - & 0.1297 \\
$\operatorname{LSD}_{\gamma=-1} \& \quad \beta=1$ & 24.59 & 3.19 & 0.21 & 0.01 & - & - & 0.1297 \\
\hline
\end{tabular}

Table 4 Estimates of the parameter for drosophila data in Table 3

\begin{tabular}{cccccccc}
\hline$\gamma \downarrow \beta \rightarrow$ & 0 & 0.2 & 0.4 & 0.6 & 0.8 & 0.9 & 1 \\
\hline-0.8 & 0.088 & 0.113 & 0.123 & 0.127 & 0.129 & 0.129 & 0.13 \\
-0.7 & 0.101 & 0.117 & 0.124 & 0.127 & 0.129 & 0.129 & 0.13 \\
-0.6 & 0.112 & 0.121 & 0.126 & 0.128 & 0.129 & 0.129 & 0.13 \\
-0.5 & 0.125 & 0.126 & 0.127 & 0.128 & 0.129 & 0.13 & 0.13 \\
-0.4 & 0.145 & 0.132 & 0.129 & 0.129 & 0.129 & 0.13 & 0.13 \\
-0.3 & 0.177 & 0.139 & 0.131 & 0.129 & 0.129 & 0.13 & 0.13 \\
-0.2 & 0.227 & 0.151 & 0.134 & 0.13 & 0.13 & 0.13 & 0.13 \\
-0.1 & 0.291 & 0.169 & 0.137 & 0.131 & 0.13 & 0.13 & 0.13 \\
0 & 0.357 & 0.194 & 0.142 & 0.132 & 0.13 & 0.13 & 0.13 \\
0.1 & 0.417 & 0.228 & 0.148 & 0.133 & 0.13 & 0.13 & 0.13 \\
0.2 & 0.47 & 0.269 & 0.157 & 0.134 & 0.13 & 0.13 & 0.13 \\
0.3 & 0.514 & 0.311 & 0.169 & 0.135 & 0.13 & 0.13 & 0.13 \\
0.4 & 0.553 & 0.353 & 0.184 & 0.137 & 0.131 & 0.13 & 0.13 \\
0.5 & 0.586 & 0.393 & 0.204 & 0.139 & 0.131 & 0.13 & 0.13 \\
0.6 & 0.615 & 0.43 & 0.226 & 0.142 & 0.131 & 0.13 & 0.13 \\
0.7 & 0.641 & 0.463 & 0.252 & 0.145 & 0.131 & 0.13 & 0.13 \\
0.8 & 0.663 & 0.494 & 0.278 & 0.149 & 0.131 & 0.13 & 0.13 \\
\hline
\end{tabular}

(a) All the estimators corresponding to large negative values of $\gamma$ and/or values of $\beta$ close to 1 generate outlier resistant methods,

(b) Estimators corresponding to large positive values of $\gamma$ are relatively poor in terms of robustness, especially for small $\beta$

(c) All estimators for $\beta=1$ are identical, irrespective of the value of $\gamma$.

Once again this example shows that large positive values of $\gamma$ with $\beta$ close to zero are the more unstable distances within the LSD class.

\section{Testing Parametric Hypothesis using the LSD Measures}

Now we focus our attention on hypothesis testing, the other important paradigm of statistical inference.

\subsection{One Sample problem}

We consider a parametric family of densities $\mathcal{F}=\left\{f_{\theta}: \theta \in \Theta \subseteq \mathbb{R}^{p}\right\}$ as above. Suppose we are given a random sample $X_{1}, \ldots, X_{n}$ of size $n$ from the 
population. Based on this sample, we want to test the hypothesis

$$
H_{0}: \theta=\theta_{0} \text { against } H_{1}: \theta \neq \theta_{0} .
$$

When the model is correctly specified and the null hypothesis is correct, $f_{\theta_{0}}$ is the data generating density. We consider the test statistics based on the LSD with parameter $\beta$ and $\gamma$ as follows:

$$
W_{\beta, \gamma}\left(\hat{\theta}_{\beta, \gamma}, \theta_{0}\right)=2 n \operatorname{LSD}_{\beta, \gamma}\left(f_{\hat{\theta}_{\beta, \gamma}}, f_{\theta_{0}}\right),
$$

where $\operatorname{LSD}_{\beta, \gamma}\left(f_{\hat{\theta}_{\beta, \gamma}}, f_{\theta_{0}}\right)$ has the form given in (9).

Theorem 6. The asymptotic distribution of the test statistic $W_{\beta, \gamma}\left(f_{\hat{\theta}_{\beta, \gamma}}, f_{\theta_{0}}\right)$, under the null hypothesis $H_{0}: \theta=\theta_{0}$, coincides with the distribution of

$$
\sum_{i=1}^{r} \zeta_{i}^{\beta}\left(\theta_{0}\right) Z_{i}^{2}
$$

where $Z_{1}, \ldots, Z_{r}$ are independent standard normal variables, $\zeta_{1}^{\beta}\left(\theta_{0}\right), \ldots$, $\zeta_{r}^{\beta}\left(\theta_{0}\right)$ are the nonzero eigenvalues of $A_{\beta}\left(\theta_{0}\right) J_{\beta}^{-1}\left(\theta_{0}\right) K_{\beta}\left(\theta_{0}\right) J_{\beta}^{-1}\left(\theta_{0}\right)$, with $J_{\beta}(\cdot)$ and $K_{\beta}(\cdot)$ as defined in Theorem 5 and the matrix $A_{\beta}\left(\theta_{0}\right)$ is defined as

$$
A_{\beta}\left(\theta_{0}\right)=\left.\nabla\left[\nabla \operatorname{LSD}_{\beta, \gamma}\left(f_{\theta}, f_{\theta_{0}}\right)\right]\right|_{\theta=\theta_{0}}
$$

and

$$
r=\operatorname{rank}\left(J_{\beta}^{-1}\left(\theta_{0}\right) K_{\beta}\left(\theta_{0}\right) J_{\beta}^{-1}\left(\theta_{0}\right) A_{\beta}\left(\theta_{0}\right) J_{\beta}^{-1}\left(\theta_{0}\right) K_{\beta}\left(\theta_{0}\right) J_{\beta}^{-1}\left(\theta_{0}\right)\right) .
$$

Proof. We consider the second order Taylor series expansion of $\operatorname{LSD}_{\beta, \gamma}\left(f_{\theta}, f_{\theta_{0}}\right)$ around $\theta=\theta_{0}$ at $\theta=\hat{\theta}_{\beta}$ as,

$$
\begin{aligned}
\operatorname{LSD}_{\beta, \gamma}\left(f_{\hat{\theta}_{\beta}}, f_{\theta_{0}}\right)= & \operatorname{LSD}_{\beta, \gamma}\left(f_{\theta_{0}}, f_{\theta_{0}}\right)+\left.\sum_{i=1}^{p} \nabla_{i} \operatorname{LSD}_{\beta, \gamma}\left(f_{\theta}, f_{\theta_{0}}\right)\right|_{\theta=\theta_{0}}\left(\hat{\theta}_{\beta}^{i}-\theta_{0}^{i}\right) \\
& +\left.\frac{1}{2} \sum_{i, j} \nabla_{i j} \operatorname{LSD}_{\beta, \gamma}\left(f_{\theta}, f_{\theta_{0}}\right)\right|_{\theta=\theta_{0}}\left(\hat{\theta}_{\beta}^{i}-\theta_{0}^{i}\right)\left(\hat{\theta}_{\beta}^{j}-\theta_{0}^{j}\right) \\
& +o\left(\left\|\hat{\theta}_{\beta}-\theta_{0}\right\|^{2}\right),
\end{aligned}
$$

where $\nabla_{i}$ and $\nabla_{i j}$ represent the indicated partial derivatives with respect to the components of $\theta$. Now we have

$$
\operatorname{LSD}_{\beta, \gamma}\left(f_{\theta_{0}}, f_{\theta_{0}}\right)=0
$$

and

$$
\left.\nabla_{i} \operatorname{LSD}_{\beta, \gamma}\left(f_{\theta}, f_{\theta_{0}}\right)\right|_{\theta=\theta_{0}}=0
$$

Note that the above second order partial derivative of $\operatorname{LSD}_{\beta, \gamma}\left(f_{\theta}, f_{\theta_{0}}\right)$ at $\theta=\theta_{0}$ is independent of $\gamma$ and so we will denote that as function of $\beta$ only. We will denote the second order partial derivatives of $\operatorname{LSD}_{\beta, \gamma}\left(f_{\theta}, f_{\theta_{0}}\right)$ in $(22)$ by 
$a_{i j}^{\beta}\left(\theta_{0}\right)$. Also denote $A_{\beta}\left(\theta_{0}\right)=\left(a_{i j}^{\beta}\left(\theta_{0}\right)\right)_{i, j=1, \ldots, p}$. Now from the above Taylor series expansion it is clear that the random variables

$$
\begin{array}{r}
W_{\beta, \gamma}\left(\hat{\theta}_{\beta, \gamma}, \theta_{0}\right)=2 n \operatorname{LSD}_{\beta, \gamma}\left(f_{\hat{\theta}_{\beta, \gamma}}, f_{\theta_{0}}\right) \\
\text { and } \quad \sqrt{n}\left(\hat{\theta}_{\beta, \gamma}-\theta_{0}\right)^{T} A_{\beta}\left(\theta_{0}\right) \sqrt{n}\left(\hat{\theta}_{\beta, \gamma}-\theta_{0}\right)
\end{array}
$$

have the same asymptotic distribution. Now we know from the previous section that the asymptotic distribution of $\sqrt{n}\left(\hat{\theta}_{\beta, \gamma}-\theta_{0}\right)$ is normal with mean zero and variance $J_{\beta}^{-1}\left(\theta_{0}\right) K_{\beta}\left(\theta_{0}\right) J_{\beta}^{-1}\left(\theta_{0}\right)$. Further we know that for $X \sim N_{q}(0, \Sigma)$, and a $q$-dimensional real symmetric matrix $A$, the distribution of the quadratic form $X^{T} A X$ is the same as that of $\sum_{i=1}^{r} \zeta_{i}^{\beta} Z_{i}^{2}$, where $Z_{1}, \ldots, Z_{r}$ are independent standard normal variables, $r=\operatorname{rank}(\Sigma A \Sigma), r \geq 1$ and $\zeta_{1}^{\beta}, \ldots, \zeta_{r}^{\beta}$ are the nonzero eigenvalues of $A \Sigma$ (Dik and Gunst, 1985, Corollary 2.1). Applying this result with $X=\sqrt{n}\left(\hat{\theta}_{\beta, \gamma}-\theta_{0}\right)$ the theorem is established. It is evident that the asymptotic distribution of the statistic depends on $\beta$ only and is independent of $\gamma$.

Theorem 7. An approximation to the power function of the test statistic $W_{\beta, \gamma}\left(\hat{\theta}_{\beta, \gamma}, \theta_{0}\right)$ for testing $H_{0}: \theta=\theta_{0}$ against $H_{1}: \theta \neq \theta_{0}$ at the significance level $\alpha$ is given by

$$
\pi_{n, \alpha}^{\beta, \gamma}\left(\theta^{*}\right)=1-\Phi\left(\frac{\sqrt{n}}{\sigma_{\beta, \gamma}\left(\theta^{*}\right)}\left(\frac{t_{\alpha}^{\beta, \gamma}}{2 n}-\operatorname{LSD}_{\beta, \gamma}\left(f_{\theta^{*}}, f_{\theta_{0}}\right)\right)\right), \quad \theta^{*} \neq \theta_{0}
$$

where $t_{\alpha}^{\beta, \gamma}$ is the $(1-\alpha)^{\text {th }}$ quantile of the asymptotic distribution of $W_{\beta, \gamma}\left(\hat{\theta}_{\beta, \gamma}, \theta_{0}\right)$, and $\sigma_{\beta, \gamma}\left(\theta^{*}\right)$ is defined as

$$
\sigma_{\beta, \gamma}^{2}(\theta)=M_{\beta, \gamma}(\theta)^{T} J_{\beta}^{-1}(\theta) K_{\beta}(\theta) J_{\beta}^{-1}(\theta) M_{\beta, \gamma}(\theta)
$$

with

$$
M_{\beta, \gamma}(\theta)=\nabla \operatorname{LSD}_{\beta, \gamma}\left(f_{\theta}, f_{\theta_{0}}\right) .
$$

Proof. Fix some $\theta^{*} \neq \theta_{0}$. Consider the first order Taylor series expansion of $\operatorname{LSD}_{\beta, \gamma}\left(f_{\hat{\theta}_{\beta, \gamma}}, f_{\theta_{0}}\right)$ under $f_{\theta^{*}}$ as

$\operatorname{LSD}_{\beta, \gamma}\left(f_{\hat{\theta}_{\beta, \gamma}}, f_{\theta_{0}}\right)=\operatorname{LSD}_{\beta, \gamma}\left(f_{\theta^{*}}, f_{\theta_{0}}\right)+M_{\beta, \gamma}\left(\theta^{*}\right)^{T}\left(\hat{\theta}_{\beta, \gamma}-\theta^{*}\right)+o\left(\left\|\hat{\theta}_{\beta, \gamma}-\theta^{*}\right\|\right)$ where $M_{\beta, \gamma}$ is as defined in the theorem. Now we know that, under $\theta^{*}$,

$$
\sqrt{n}\left(\hat{\theta}_{\beta, \gamma}-\theta^{*}\right) \rightarrow N\left(0, J_{\beta}^{-1}\left(\theta^{*}\right) K_{\beta}\left(\theta^{*}\right) J_{\beta}^{-1}\left(\theta^{*}\right)\right) \text { as } n \rightarrow \infty
$$

and $\sqrt{n} o\left(\left\|\hat{\theta}_{\beta, \gamma}-\theta^{*}\right\|\right)=o_{p}(1)$. Thus we get that the random variables

$\sqrt{n}\left[\operatorname{LSD}_{\beta, \gamma}\left(f_{\hat{\theta}_{\beta, \gamma}}, f_{\theta_{0}}\right)-\operatorname{LSD}_{\beta, \gamma}\left(f_{\theta^{*}}, f_{\theta_{0}}\right)\right] \quad$ and $\quad M_{\beta, \gamma}\left(\theta^{*}\right)^{T} \sqrt{n}\left(\hat{\theta}_{\beta, \gamma}-\theta^{*}\right)$

have the same asymtotic distribution. Therefore, we have

$$
\sqrt{n}\left[\operatorname{LSD}_{\beta, \gamma}\left(f_{\hat{\theta}_{\beta, \gamma}}, f_{\theta_{0}}\right)-\operatorname{LSD}_{\beta, \gamma}\left(f_{\theta^{*}}, f_{\theta_{0}}\right)\right] \rightarrow N\left(0, \sigma_{\beta, \gamma}\left(\theta^{*}\right)\right)
$$

where $\sigma_{\beta, \gamma}\left(\theta^{*}\right)$ is as given in (24) above. Hence the desired approximation to the power function follows from the above asymptotic distribution. 
6.2 Two-Sample Problem

Again consider a parametric family of densities $\left\{f_{\theta}: \theta \in \Theta \subseteq \mathbb{R}^{p}\right\}$ as above in one sample problem, but here we are given two random samples $X_{1}, \ldots, X_{n}$ of size $n$ and $Y_{1}, \ldots, Y_{m}$ of size $m$ from two distributions within the model having parameters $\theta_{1}$ and $\theta_{2}$ respectively and based on these two samples, we want to test for the homogeneity of the two samples, i.e. to test the hypothesis

$$
H_{0}: \theta_{1}=\theta_{2} \text { against } \quad H_{1}: \theta_{1} \neq \theta_{2} \text {. }
$$

We will consider the estimator ${ }^{(1)} \hat{\theta}_{\beta, \gamma}$ and ${ }^{(2)} \hat{\theta}_{\beta, \gamma}$ of $\theta_{1}$ and $\theta_{2}$ respectively, obtained by minimizing the LSD having parameters $\beta, \gamma$ and, as before, will consider the test statistic based on the LSD with parameter $\beta$ and $\gamma$ as given by

$$
S_{\beta, \gamma}\left({ }^{(1)} \hat{\theta}_{\beta, \gamma},{ }^{(2)} \hat{\theta}_{\beta, \gamma}\right)=\frac{2 n m}{n+m} \operatorname{LSD}_{\beta, \gamma}\left(f_{(1) \hat{\theta}_{\beta, \gamma}}, f_{(2) \hat{\theta}_{\beta, \gamma}}\right) .
$$

Now, first let us consider the asymptotic distribution of the test statistic $S_{\beta, \gamma}\left({ }^{(1)} \hat{\theta}_{\beta, \gamma},{ }^{(2)} \hat{\theta}_{\beta, \gamma}\right)$ under $H_{0}$ in the following theorem. Assume that $\frac{m}{m+n} \rightarrow$ $\omega,(0<\omega<1)$, as $m \rightarrow \infty$ and $n \rightarrow \infty$.

Theorem 8. The asymptotic distribution of the test statistic $S_{\beta, \gamma}\left({ }^{(1)} \hat{\theta}_{\beta, \gamma},{ }^{(2)} \hat{\theta}_{\beta, \gamma}\right)$, under the null hypothesis $H_{0}: \theta_{1}=\theta_{2}$, coincides with the distribution of

$$
\sum_{i=1}^{r} \zeta_{i}^{\beta}\left(\theta_{1}\right) Z_{i}^{2}
$$

where $Z_{1}, \ldots, Z_{r}$ are independent standard normal variables, $\zeta_{1}^{\beta}\left(\theta_{1}\right), \ldots$, $\zeta_{r}^{\beta}\left(\theta_{1}\right)$ are the nonzero eigenvalues of $A_{\beta}\left(\theta_{1}\right) J_{\beta}^{-1}\left(\theta_{1}\right) K_{\beta}\left(\theta_{1}\right) J_{\beta}^{-1}\left(\theta_{1}\right)$, with $J_{\beta}(\cdot), K_{\beta}(\cdot)$ and $A_{\beta}(\cdot)$ as defined in the previous section and

$$
r=\operatorname{rank}\left(J_{\beta}^{-1}\left(\theta_{1}\right) K_{\beta}\left(\theta_{1}\right) J_{\beta}^{-1}\left(\theta_{1}\right) A_{\beta}\left(\theta_{1}\right) J_{\beta}^{-1}\left(\theta_{1}\right) K_{\beta}\left(\theta_{1}\right) J_{\beta}^{-1}\left(\theta_{1}\right)\right) .
$$

Proof. We have

$$
\sqrt{n}\left({ }^{(1)} \hat{\theta}_{\beta, \gamma}-\theta_{1}\right) \rightarrow N\left(0, J_{\beta}^{-1}\left(\theta_{1}\right) K_{\beta}\left(\theta_{1}\right) J_{\beta}^{-1}\left(\theta_{1}\right)\right)
$$

and

$$
\sqrt{m}\left({ }^{(2)} \hat{\theta}_{\beta, \gamma}-\theta_{2}\right) \rightarrow N\left(0, J_{\beta}^{-1}\left(\theta_{2}\right) K_{\beta}\left(\theta_{2}\right) J_{\beta}^{-1}\left(\theta_{2}\right)\right) .
$$

Let $\frac{m}{m+n} \rightarrow \omega \in(0,1)$ as $m, n \rightarrow \infty$. Then we have

$$
\sqrt{\frac{m n}{m+n}}\left({ }^{(1)} \hat{\theta}_{\beta, \gamma}-\theta_{1}\right) \rightarrow N\left(0, \omega J_{\beta}^{-1}\left(\theta_{1}\right) K_{\beta}\left(\theta_{1}\right) J_{\beta}^{-1}\left(\theta_{1}\right)\right)
$$

and

$$
\sqrt{\frac{m n}{m+n}}\left({ }^{(2)} \hat{\theta}_{\beta, \gamma}-\theta_{2}\right) \rightarrow N\left(0,(1-\omega) J_{\beta}^{-1}\left(\theta_{2}\right) K_{\beta}\left(\theta_{2}\right) J_{\beta}^{-1}\left(\theta_{2}\right)\right)
$$


Now, under $H_{0}: \theta_{1}=\theta_{2}$, we get that

$$
\sqrt{\frac{m n}{m+n}}\left({ }^{(1)} \hat{\theta}_{\beta, \gamma}{ }^{(2)} \hat{\theta}_{\beta, \gamma}\right) \rightarrow N\left(0, J_{\beta}^{-1}\left(\theta_{1}\right) K_{\beta}\left(\theta_{1}\right) J_{\beta}^{-1}\left(\theta_{1}\right)\right)
$$

Next consider the second order Taylor series expansion of $\operatorname{LSD}_{\beta, \gamma}\left(f_{\theta_{1}}, f_{\theta_{2}}\right)$ around $\theta_{1}=\theta_{2}$ at $\left({ }^{(1)} \hat{\theta}_{\beta, \gamma},{ }^{(2)} \hat{\theta}_{\beta, \gamma}\right)$ as follows

$$
\begin{aligned}
\operatorname{LSD}_{\beta, \gamma}\left(f_{(1) \hat{\theta}_{\beta, \gamma}}, f_{(2) \hat{\theta}_{\beta, \gamma}}\right)= & \frac{1}{2} \sum_{i, j=1}^{p}\left(\frac{\partial^{2} \operatorname{LSD}_{\beta, \gamma}\left(f_{\theta_{1}}, f_{\theta_{2}}\right)}{\partial \theta_{1 i} \partial \theta_{1 j}}\right)_{\theta_{1}=\theta_{2}} \\
& \left(\hat{\theta}_{\beta, \gamma}^{1 i}-\theta_{1 i}\right)\left(\hat{\theta}_{\beta, \gamma}^{1 j}-\theta_{1 j}\right) \\
& +\sum_{i, j=1}^{p}\left(\frac{\partial^{2} \mathrm{LSD}_{\beta, \gamma}\left(f_{\theta_{1}}, f_{\theta_{2}}\right)}{\partial \theta_{1 i} \partial \theta_{2 j}}\right)_{\theta_{1}=\theta_{2}} \\
& \left(\hat{\theta}_{\beta, \gamma}^{1 i}-\theta_{1 i}\right)\left(\hat{\theta}_{\beta, \gamma}^{2 j}-\theta_{2 j}\right) \\
& +\frac{1}{2} \sum_{i, j=1}^{p}\left(\frac{\partial^{2} \mathrm{LSD}_{\beta, \gamma}\left(f_{\theta_{1}}, f_{\theta_{2}}\right)}{\partial \theta_{2 i} \partial \theta_{2 j}}\right)_{\theta_{1}=\theta_{2}} \\
& \left(\hat{\theta}_{\beta, \gamma}^{2 i}-\theta_{2 i}\right)\left(\hat{\theta}_{\beta, \gamma}^{2 j}-\theta_{2 j}\right) \\
& +o\left(\left\|{ }^{(1)} \hat{\theta}_{\beta, \gamma}-\theta_{1}\right\|^{2}\right)+o\left(\left\|\hat{\theta}_{\beta, \gamma}-\theta_{2}\right\|^{2}\right) .
\end{aligned}
$$

But for $i=1, \ldots, p$, we have

$$
\frac{\partial \operatorname{LSD}_{\beta, \gamma}\left(f_{\theta_{1}}, f_{\theta_{2}}\right)}{\partial \theta_{1 i}}=\frac{1+\beta}{B}\left[\frac{\sum f_{\theta_{1 i}}^{1+\beta} u_{\theta_{1 i}}}{\sum f_{\theta_{1 i}}^{1+\beta}}-\frac{\sum f_{\theta_{1 i}}^{A} f_{\theta_{2}}^{B} u_{\theta_{1 i}}}{\sum f_{\theta_{1 i}}^{A} f_{\theta_{2}}^{B}}\right] .
$$

where $B=\beta-\gamma(1-\beta)$ and $A=1+\gamma(1-\beta)$ and hence

$$
\begin{aligned}
& \left(\frac{\partial^{2} \operatorname{LSD}_{\beta, \gamma}\left(f_{\theta_{1}}, f_{\theta_{2}}\right)}{\partial \theta_{1 i} \partial \theta_{1 j}}\right)_{\theta_{1}=\theta_{2}}=a_{i j}^{\beta}\left(\theta_{1}\right), \\
& \left(\frac{\partial^{2} \operatorname{LSD}_{\beta, \gamma}\left(f_{\theta_{1}}, f_{\theta_{2}}\right)}{\partial \theta_{1 i} \partial \theta_{2 j}}\right)_{\theta_{1}=\theta_{2}}=-a_{i j}^{\beta}\left(\theta_{1}\right), \\
& \left(\frac{\partial^{2} \operatorname{LSD}_{\beta, \gamma}\left(f_{\theta_{1}}, f_{\theta_{2}}\right)}{\partial \theta_{2 i} \partial \theta_{2 j}}\right)_{\theta_{1}=\theta_{2}}=a_{i j}^{\beta}\left(\theta_{1}\right) .
\end{aligned}
$$


As in the one sample case, here also the second order partial derivatives depend on $\beta$ only. Therefore, we get

$$
\begin{aligned}
2 \operatorname{LSD}_{\beta, \gamma}\left(f_{(1)} \hat{\theta}_{\beta, \gamma}, f_{(2)} \hat{\theta}_{\beta, \gamma}\right)= & \left({ }^{(1)} \hat{\theta}_{\beta, \gamma}-\theta_{1}\right)^{T} A_{\beta}\left(\theta_{1}\right)\left({ }^{(1)} \hat{\theta}_{\beta, \gamma}-\theta_{1}\right) \\
& -2\left({ }^{(1)} \hat{\theta}_{\beta, \gamma}-\theta_{1}\right)^{T} A_{\beta}\left(\theta_{1}\right)\left({ }^{(2)} \hat{\theta}_{\beta, \gamma}-\theta_{1}\right) \\
& +\left({ }^{(2)} \hat{\theta}_{\beta, \gamma}-\theta_{1}\right)^{T} A_{\beta}\left(\theta_{1}\right)\left({ }^{(2)} \hat{\theta}_{\beta, \gamma}-\theta_{1}\right) \\
& +o\left(\|\|^{(1)} \hat{\theta}_{\beta, \gamma}-\theta_{1} \|^{2}\right)+o\left(\left\|{ }^{(2)} \hat{\theta}_{\beta, \gamma}-\theta_{2}\right\|^{2}\right) \\
= & \left({ }^{(1)} \hat{\theta}_{\beta, \gamma}-{ }^{(2)} \hat{\theta}_{\beta, \gamma}\right)^{T} A_{\beta}\left(\theta_{1}\right)\left({ }^{(1)} \hat{\theta}_{\beta, \gamma}-{ }^{(2)} \hat{\theta}_{\beta, \gamma}\right) \\
& +o\left(\left\|{ }^{(1)} \hat{\theta}_{\beta, \gamma}-\theta_{1}\right\|^{2}\right)+o\left(\left\|{ }^{(2)} \hat{\theta}_{\beta, \gamma}-\theta_{2}\right\|^{2}\right),
\end{aligned}
$$

with

$$
o\left(\left\|{ }^{(1)} \hat{\theta}_{\beta, \gamma}-\theta_{1}\right\|^{2}\right)=o_{p}\left(\frac{1}{n}\right) \quad \text { and } \quad o\left(\left\|{ }^{(2)} \hat{\theta}_{\beta, \gamma}-\theta_{2}\right\|^{2}\right)=o_{p}\left(\frac{1}{m}\right) .
$$

Thus the asymptotic distribution of

$$
S_{\beta, \gamma}\left({ }^{(1)} \hat{\theta}_{\beta, \gamma}{ }^{(2)} \hat{\theta}_{\beta, \gamma}\right)=\frac{2 n m}{n+m} \operatorname{LSD}_{\beta, \gamma}\left(f_{(1) \hat{\theta}_{\beta, \gamma}}, f_{(2) \hat{\theta}_{\beta, \gamma}}\right)
$$

coincides with the distribution of the random variable $\sum_{i=1}^{r} \zeta_{i}^{\beta}\left(\theta_{1}\right) Z_{i}^{2}$. Like the one sample case the asymptotic distribution depends on $\beta$ only.

We have noted that the asymptotic distribution of the LSD based test statistics under the simple null hypothesis is independent of the parameter $\gamma$. Maji, Ghosh and Basu (2014) have also reported a similar observation for the robustness of the corresponding test statistics. They have shown that the first order influence function of the test statistics is always zero at the simple null and its second order influence function under null, being a quadratic form in the first order influence function of the minimum LSD estimator used, is independent of the parameter $\gamma$. However, the numerical illustrations reported in their paper and in the next section of present paper, this independence is not true for samples with moderate size. Therefore, as in the case of estimation, the robustness of the LSD based test of simple null hypothesis can not e indicated in terms of the influence function analysis even if we even go up to second order. However, Maji, Ghosh and Basu (2014) showed that the robustness of the minimum LSD estimators can be measured quite accurately in terms of the secord order influence function of the estimator. Extending the same idea in case of testing, it is a routine exercise to see that the third order influence function of the test statistics at the null, being a function of the second order influence function of the corresponding estimator, can serve a better measure of robustness in this case. In this article we have restricted ourselves to the simple null case. However the results may be extended to the case involving nuisance parameters following the same general approach. 


\section{A Two-Sample Example}

Here we will discuss a two sample real data example which is known to give rise to occasional spurious counts. This experiment is available in Woodruff et al. (1984) and has been analyzed previously by Simpson (1989). This is a sexlinked recessive lethal experiment in drosophila (fruit flies) to test chemical mutagenicity. Male flies were exposed either to $2000 \mu \mathrm{g}$ butyraldehyde or to control conditions. The responses are the numbers of recessive lethal mutations observed among daughters of these flies. The data are given in Table 5. We will use a Poisson model in this experiment where the control responses are supposed to follow Poisson distribution with mean $\theta_{0}$ and and the treated responses follows a Poisson distribution with mean $\theta_{1}$. The two large counts for the treated group appears to be possible outliers. We want to test $H_{0}: \theta_{0} \geq \theta_{1}$ against $H_{1}: \theta_{0}<\theta_{1}$. The test statistic for testing this hypothesis is given by,

$$
{ }^{*} S_{\beta, \gamma}\left({ }^{(1)} \hat{\theta}_{\beta, \gamma},{ }^{(2)} \hat{\theta}_{\beta, \gamma}\right)=\frac{1}{\zeta\left({ }^{(0)} \hat{\theta}_{\beta, \gamma}\right)} \frac{2 n m}{n+m} \operatorname{LSD}_{\beta, \gamma}\left(p\left({ }^{(1)} \hat{\theta}_{\beta, \gamma}\right), p\left({ }^{(2)} \hat{\theta}_{\beta, \gamma}\right)\right),
$$

where

$$
\zeta\left({ }^{(0)} \hat{\theta}_{\beta, \gamma}\right)=\frac{A_{\beta, \gamma}\left({ }^{(0)} \hat{\theta}_{\beta, \gamma}\right) K_{\beta, \gamma}\left({ }^{(0)} \hat{\theta}_{\beta, \gamma}\right)}{J_{\beta, \gamma}^{2}\left({ }^{(0)} \hat{\theta}_{\beta, \gamma}\right)} .
$$

The asymptotic distribution of the statistic ${ }^{*} S_{\gamma, \lambda}\left({ }^{(1)} \hat{\theta}_{\beta},{ }^{(2)} \hat{\theta}_{\beta}\right)$ is chi-square with one degree of freedom and the corresponding $p$-values are calculated and shown in the tables. The statistic for $\beta=\gamma=0$ case is not same as the likelihood ratio statistic but they are asymptotically same. Though the tests are different but the non-robust nature of the likelihood test can be seen under this set-up also. The results are shown in Tables 6 and 7. From the results it is evident that for $\beta \geq 0.6$ and irrespective of $\gamma$ the presence and the absence of outliers has little impact on $p$-values. For $\gamma<0$, lower values of $\beta$ also give close $p$-values but for $\gamma>0$, lower values of $\beta$ the method does not perform well. It is clear that for large values of $\beta$ (say $\geq 0.5$ ), the full data and the outlier deleted data basically lead to the same conclusion and almost identical $p$-values irrespective of the value of $\gamma$. The situation changes when $\beta$ is a small positive value close to 0 . In this case the role of $\gamma$ becomes decisive. Large positive values of $\gamma$ and small values of $\beta$ lead to a highly unstable results. The outlier deleted $p$-values and full data $p$-values are far from close in these cases. However the negative values of $\gamma$ lead to stable inference even when $\beta=0$ or in its neighborhood. On the whole it appears that the two large counts in the treated group indicate a false significance for the likelihood ratio test and some other members of our class, but the more robust members clearly recognize the significance to be false. 
Table 5 Frequencies of the number of recessive lethal daughters for drosophila data

\begin{tabular}{ccccccccc}
\hline$x$ & 0 & 1 & 2 & 3 & 4 & 5 & 6 & 7 \\
\hline Observed (Control) & 159 & 15 & 3 & 0 & 0 & 0 & 0 & 0 \\
Observed (Treated) & 110 & 11 & 5 & 0 & 0 & 0 & 1 & 1 \\
\hline
\end{tabular}

Table 6 Estimated Poisson parameters for the two-sample drosophila example; the numbers within the parentheses show the corresponding estimates for the treated case after deleting the two outliers. The parameter $\gamma$ is held at 0 for the first part and the parameter $\beta$ is held at 0 for the second part.

\begin{tabular}{cccccc}
\hline$\beta$ & 0.2 & 0.4 & 0.6 & 0.8 & 1 \\
\hline${ }^{(1)} \hat{\theta}_{\beta}$ & 0.1091 & 0.1027 & 0.099 & 0.0969 & 0.0957 \\
${ }^{(2)} \hat{\theta}_{\beta}$ & 0.153 & 0.1266 & 0.1143 & 0.1077 & 0.1042 \\
& $(0.1432)$ & $(0.1255)$ & $(0.1141)$ & $(0.1105)$ & $(0.1057)$ \\
${ }^{(0)} \hat{\theta}_{\beta}$ & 0.1264 & 0.1122 & 0.1051 & 0.1029 & 0.1 \\
& $(0.1229)$ & $(0.1118)$ & $(0.105)$ & $(0.1028)$ & $(0.1)$ \\
\hline$\gamma$ & 0.2 & 0.4 & 0.6 & 0.8 & 1 \\
\hline${ }^{(1)} \hat{\theta}_{\gamma}$ & 0.1216 & 0.1245 & 0.1273 & 0.1303 & 0.133 \\
${ }^{(2)} \hat{\theta}_{\gamma}$ & 0.3227 & 0.4139 & 0.4916 & 0.5547 & 0.6059 \\
& $(0.1763)$ & $(0.1854)$ & $(0.1938)$ & $(0.2015)$ & $(0.2084)$ \\
${ }^{(0)} \hat{\theta}_{\gamma}$ & 0.2182 & 0.2851 & 0.3497 & 0.4055 & 0.4521 \\
& $(0.1444)$ & $(0.1501)$ & $(0.1555)$ & $(0.1607)$ & $(0.1655)$ \\
\hline
\end{tabular}

Table 7 The $p$-values for the two-sample drosophila data. The outlier deleted $p$-values are given in the second line of each block

\begin{tabular}{|c|c|c|c|c|c|c|c|c|c|c|c|}
\hline$\gamma \downarrow \beta \rightarrow$ & 0 & 0.1 & 0.2 & 0.3 & 0.4 & 0.5 & 0.6 & 0.7 & 0.8 & 0.9 & 1 \\
\hline \multirow[t]{2}{*}{-0.8} & 0.262 & 0.341 & 0.426 & 0.509 & 0.582 & 0.642 & 0.695 & 0.738 & 0.775 & 0.806 & 0.831 \\
\hline & 0.264 & 0.343 & 0.428 & 0.511 & 0.583 & 0.643 & 0.696 & 0.739 & 0.776 & 0.807 & 0.832 \\
\hline \multirow[t]{2}{*}{-0.7} & 0.235 & 0.325 & 0.417 & 0.506 & 0.582 & 0.644 & 0.698 & 0.74 & 0.776 & 0.807 & 0.831 \\
\hline & 0.239 & 0.327 & 0.42 & 0.508 & 0.584 & 0.646 & 0.7 & 0.741 & 0.777 & 0.807 & 0.832 \\
\hline \multirow{2}{*}{-0.6} & 0.218 & 0.314 & 0.412 & 0.501 & 0.579 & 0.645 & 0.699 & 0.742 & 0.779 & 0.807 & 0.831 \\
\hline & 0.221 & 0.317 & 0.414 & 0.503 & 0.581 & 0.647 & 0.7 & 0.744 & 0.78 & 0.808 & 0.832 \\
\hline \multirow[t]{2}{*}{-0.5} & 0.207 & 0.306 & 0.406 & 0.499 & 0.578 & 0.646 & 0.7 & 0.745 & 0.779 & 0.807 & 0.831 \\
\hline & 0.211 & 0.309 & 0.408 & 0.5 & 0.58 & 0.647 & 0.701 & 0.746 & 0.78 & 0.808 & 0.832 \\
\hline \multirow[t]{2}{*}{-0.4} & 0.199 & 0.301 & 0.402 & 0.496 & 0.579 & 0.647 & 0.704 & 0.746 & 0.782 & 0.809 & 0.831 \\
\hline & 0.208 & 0.305 & 0.404 & 0.497 & 0.581 & 0.649 & 0.705 & 0.747 & 0.783 & 0.81 & 0.832 \\
\hline \multirow[t]{2}{*}{-0.3} & 0.187 & 0.294 & 0.398 & 0.494 & 0.578 & 0.648 & 0.702 & 0.747 & 0.782 & 0.81 & 0.831 \\
\hline & 0.211 & 0.303 & 0.403 & 0.498 & 0.58 & 0.649 & 0.704 & 0.749 & 0.783 & 0.811 & 0.832 \\
\hline \multirow[t]{2}{*}{-0.2} & 0.149 & 0.284 & 0.395 & 0.493 & 0.578 & 0.647 & 0.705 & 0.749 & 0.785 & 0.81 & 0.831 \\
\hline & 0.22 & 0.307 & 0.402 & 0.496 & 0.58 & 0.648 & 0.706 & 0.75 & 0.786 & 0.811 & 0.832 \\
\hline \multirow[t]{2}{*}{-0.1} & 0.058 & 0.251 & 0.388 & 0.491 & 0.576 & 0.648 & 0.705 & 0.749 & 0.785 & 0.811 & 0.831 \\
\hline & 0.235 & 0.315 & 0.404 & 0.497 & 0.579 & 0.651 & 0.706 & 0.75 & 0.786 & 0.812 & 0.832 \\
\hline \multirow[t]{2}{*}{0} & 0.003 & 0.156 & 0.367 & 0.488 & 0.575 & 0.649 & 0.707 & 0.753 & 0.786 & 0.811 & 0.831 \\
\hline & 0.141 & 0.328 & 0.411 & 0.496 & 0.578 & 0.65 & 0.708 & 0.754 & 0.787 & 0.812 & 0.832 \\
\hline \multirow[t]{2}{*}{0.1} & 0 & 0.03 & 0.306 & 0.479 & 0.575 & 0.648 & 0.707 & 0.753 & 0.788 & 0.814 & 0.831 \\
\hline & 0.293 & 0.345 & 0.419 & 0.5 & 0.579 & 0.65 & 0.708 & 0.754 & 0.789 & 0.814 & 0.832 \\
\hline \multirow[t]{2}{*}{0.2} & 0 & 0.001 & 0.172 & 0.454 & 0.572 & 0.648 & 0.707 & 0.754 & 0.788 & 0.814 & 0.831 \\
\hline & 0.338 & 0.369 & 0.429 & 0.504 & 0.58 & 0.651 & 0.708 & 0.755 & 0.789 & 0.815 & 0.832 \\
\hline \multirow[t]{2}{*}{0.3} & 0 & 0 & 0.037 & 0.391 & 0.566 & 0.648 & 0.708 & 0.754 & 0.79 & 0.815 & 0.831 \\
\hline & 0.392 & 0.398 & 0.444 & 0.51 & 0.583 & 0.65 & 0.709 & 0.755 & 0.791 & 0.815 & 0.832 \\
\hline \multirow[t]{2}{*}{0.4} & 0 & 0 & 0.002 & 0.261 & 0.55 & 0.647 & 0.709 & 0.755 & 0.79 & 0.815 & 0.831 \\
\hline & 0.46 & 0.435 & 0.462 & 0.517 & 0.585 & 0.652 & 0.71 & 0.756 & 0.791 & 0.816 & 0.832 \\
\hline \multirow[t]{2}{*}{0.5} & 0 & 0 & 0 & 0.101 & 0.513 & 0.644 & 0.709 & 0.757 & 0.792 & 0.816 & 0.831 \\
\hline & 0.54 & 0.478 & 0.483 & 0.526 & 0.588 & 0.652 & 0.71 & 0.758 & 0.793 & 0.817 & 0.832 \\
\hline \multirow[t]{2}{*}{0.6} & 0 & 0 & 0 & 0.019 & 0.436 & 0.639 & 0.709 & 0.758 & 0.793 & 0.816 & 0.831 \\
\hline & 0.629 & 0.528 & 0.508 & 0.539 & 0.592 & 0.653 & 0.711 & 0.759 & 0.794 & 0.817 & 0.832 \\
\hline \multirow[t]{2}{*}{0.7} & 0 & 0 & 0 & 0.002 & 0.3 & 0.629 & 0.709 & 0.757 & 0.793 & 0.817 & 0.831 \\
\hline & 0.727 & 0.584 & 0.538 & 0.552 & 0.597 & 0.655 & 0.712 & 0.758 & 0.794 & 0.818 & 0.832 \\
\hline \multirow[t]{2}{*}{0.8} & 0.003 & 0 & 0 & 0 & 0.147 & 0.605 & 0.709 & 0.758 & 0.794 & 0.817 & 0.831 \\
\hline & 0.827 & 0.646 & 0.571 & 0.568 & 0.603 & 0.657 & 0.712 & 0.759 & 0.795 & 0.818 & 0.832 \\
\hline
\end{tabular}




\section{Conclusion}

Logarithmic super divergence family acts as a super family of both LPD and LDPD family. The theoretical properties of this new family of divergences have been established for discrete models and similar results under continuous set-up can be done in subsequent works.

\section{References}

1. Ali, S. M. and S. D. Silvey (1966). A general class of coefficients of divergence of one distribution from another. Journal of the Royal Statistical Society B, 28, 131-142.

2. Basu, A., I. R. Harris, N. L. Hjort, and M. C. Jones (1998). Robust and efficient estimation by minimising a density power divergence. Biometrika, 85, 549-559.

3. Basu, A., H. Shioya, and C. Park (2011). Statistical Inference: The Minimum Distance Approach. Chapman \& Hall/CRC, Boca Raton, FL.

4. Beran, R. J. (1977). Minimum Hellinger distance estimates for parametric models. Annals of Statistics, 5, 445-463.

5. Bregman, L. M. (1967). The relaxation method of finding the common point of convex sets and its application to the solution of problems in convex programming. USSR Computational Mathematics and Mathematical Physics, 7, 200-217. Original article is in Zh. vychisl. Mat. mat. Fiz., 7, pp. 620-631, 1967.

6. Chung, K. L. (1974). A Course in Probability Theory. Academic Press, New York.

7. Cressie, N. and T. R. C. Read (1984). Multinomial goodness-of-fit tests. Journal of the Royal Statistical Society B, 46, 440-464.

8. Csisźar, I. (1963). Eine informations theoretische Ungleichung und ihre Anwendung auf den Beweis der Ergodizitat von Markoffschen Ketten. Publ. Math. Inst. Hungar. Acad. Sci., 3, 85-107.

9. Csisźar, I. (1967a). Information-type measures of difference of probability distributions and indirect observations. Studia Scientiarum Mathematicarum Hungarica, 2, 299-318.

10. Csisźar, I. (1967b). On topological properties of f-divergences. Studia Scientiarum Mathematicarum Hungarica, 2, 329-339.

11. Dik, J. J. and M. C. M, de Gunst (1985). The distribution of general quadratic forms in normal variables. Statistica Neerlandica, 39, 14-26.

12. Ghosh, A. (2014). Asymptotic Properties of Minimum S-Divergence Estimator for Discrete Models. Technical Report, Bayesian and Interdisciplinary Research Unit, Indian Statistical Institute, India.

13. Ghosh, A., I.R. Harris, A. Maji, A. Basu, and L. Pardo (2013). A Generalized Divergence for Statistical Inference. Technical Report, Bayesian and Interdisciplinary Research Unit, Indian Statistical Institute, India.

14. Jones, M. C., N. L. Hjort, I. R. Harris, and A. Basu (2001). A comparison of related density-based minimum divergence estimators. Biometrika, 88, 865-873.

15. Lehmann, E. L. (1983). Theory of Point Estimation. John Wiley \& Sons.

16. Lindsay, B. G. (1994). Efficiency versus robustness: The case for minimum Hellinger distance and related methods. Annals of Statistics, 22, 1081-1114.

17. Maji, A., A. Ghosh, and A. Basu (2014). The Logarithmic Super Divergence and its use in Statistical Inference. Technical Report, Bayesian and Interdisciplinary Research Unit, Indian Statistical Institute, India.

18. Maji, A., S. Chakraborty, and A. Basu (2014). Statistical Inference based on the Logarithmic Power Divergence. Technical Report, Bayesian and Interdisciplinary Research Unit, Indian Statistical Institute, India.

19. Pardo, L. (2006). Statistical Inference based on Divergences. CRC/Chapman-Hall.

20. Patra, S., A. Maji, A. Basu, and L. Pardo (2013). The Power Divergence and the Density Power Divergence Families : the Mathematical Connection. Sankhya B, 75, 16-28.

21. Pearson, K. (1900). On the criterion that a given system of deviations from the probable in the case of a correlated system of variables is such that it can be reasonably supposed to have arisen from random sampling. Philosophical Magazine, 50, 157-175. 
22. Renyi, A. (1961). On measures of entropy and information. In Proceedings of Fourth Berkeley Symposium on Mathematical Statistics and Probability, volume I, pages 547561. University of California.

23. Simpson, D. G. (1989). Hellinger deviance test: Efficiency, breakdown points, and examples. Journal of the American Statistical Association, 84, 107-113.

24. Windham, M. (1995). Robustifying model fitting. Journal of the Royal Statistical Society. Series B, 57, 599-609.

25. Woodruff, R. C., J. M. Mason, R. Valencia, and A. Zimmering (1984). Chemical mutagenesis testing in drosophila I: Comparison of positive and negative control data for sex-linked recessive lethal mutations and reciprocal translocations in three laboratories. Environmental Mutagenesis, 6, 189-202.

\section{Appendix}

Consistency Part: Consider the behavior of $\operatorname{LSD}\left(r_{n}, f_{\theta}\right)$ on a sphere $Q_{a}$ which has radius $a$ and center at $\theta^{g}$. We will show, for sufficiently small $a$, the probability tends to one that

$$
\operatorname{LSD}\left(r_{n}, f_{\theta}\right)>\operatorname{LSD}\left(r_{n}, f_{\theta^{g}}\right) \quad \text { for all } \theta \text { on the surface of } Q_{a}
$$

so that the LSD has a local minimum with respect to $\theta$ in the interior of $Q_{a}$. At a local minimum, the estimating equations must be satisfied. Therefore, for any $a>0$ sufficiently small, the minimum LSD estimating equation have a solution $\theta_{n}$ within $Q_{a}$ with probability tending to one as $n \rightarrow \infty$. Now taking Taylor series expansion of $\operatorname{LSD}\left(r_{n}, f_{\theta}\right)$ about $\theta=\theta^{g}$, we get

$$
\begin{aligned}
\operatorname{LSD}\left(r_{n}, f_{\theta^{g}}\right)-\operatorname{LSD}\left(r_{n}, f_{\theta}\right)= & -\left.\sum_{j}\left(\theta_{j}-\theta_{j}^{g}\right) \nabla_{j} \operatorname{LSD}\left(r_{n}, f_{\theta}\right)\right|_{\theta=\theta^{g}} \\
& -\left.\frac{1}{2} \sum_{j, k}\left(\theta_{j}-\theta_{j}^{g}\right)\left(\theta_{k}-\theta_{k}^{g}\right) \nabla_{j k} \operatorname{LSD}\left(r_{n}, f_{\theta}\right)\right|_{\theta=\theta^{g}} \\
& -\left.\frac{1}{6} \sum_{j, k, l}\left(\theta_{j}-\theta_{j}^{g}\right)\left(\theta_{k}-\theta_{k}^{g}\right)\left(\theta_{l}-\theta_{l}^{g}\right) \nabla_{j k l} \operatorname{LSD}\left(r_{n}, f_{\theta}\right)\right|_{\theta=\theta^{*}} \\
& =S_{1}+S_{2}+S_{3}, \quad \text { (say) }
\end{aligned}
$$

where $\theta^{*}$ lies between $\theta^{g}$ and $\theta$. We will now consider each terms one-byone.

For the Linear term $S_{1}$, we consider

$\nabla_{j} \operatorname{LSD}\left(r_{n}, f_{\theta}\right)=\frac{(1+\beta)}{A}\left[\left(\sum_{x} f_{\theta}^{1+\beta} u_{j \theta}\right)\left(\sum_{x} f_{\theta}^{1+\beta}\right)^{-1}-\left(\sum_{x} r_{n}^{A} f_{\theta}^{B} u_{j \theta}\right)\left(\sum_{x} r_{n}^{A} f_{\theta}^{B}\right)^{-1}\right]$.

To show

$$
\left.\nabla_{j} \operatorname{LSD}\left(r_{n}, f_{\theta}\right)\right|_{\theta=\theta^{g}} \stackrel{P}{\rightarrow} 0,
$$

we need to show

$$
\sum_{x} K\left(\delta_{n}^{g}(x)\right) f_{\theta^{g}}^{1+\beta}(x) u_{j \theta^{g}}(x) \stackrel{P}{\rightarrow} \sum_{x} K\left(\delta_{g}^{g}(x)\right) f_{\theta^{g}}^{1+\beta}(x) u_{j \theta^{g}}(x)
$$


and

$$
\sum_{x} K\left(\delta_{n}^{g}(x)\right) f_{\theta^{g}}^{1+\beta}(x) \stackrel{P}{\longrightarrow} \sum_{x} K\left(\delta_{g}^{g}(x)\right) f_{\theta^{g}}^{1+\beta}(x)
$$

as $n \rightarrow \infty$ and where $\delta_{n}^{g}(x)$ is the $\delta_{n}(x)$ evaluated at $\theta=\theta^{g}$. We will now show that

$$
\sum_{x} K\left(\delta_{n}^{g}(x)\right) f_{\theta^{g}}^{1+\beta}(x) u_{j \theta^{g}}(x) \stackrel{P}{\rightarrow} \sum_{x} K\left(\delta_{g}^{g}(x)\right) f_{\theta^{g}}^{1+\beta}(x) u_{j \theta^{g}}(x)
$$

as $n \rightarrow \infty$. Note that by assumption 7 and the fact that $r_{n}(x) \rightarrow g(x)$ almost surely (a.s.) by Strong law of large numbers (SLLN), it follows that

$$
\left|K^{\prime}(\delta)\right|=|A||\delta|^{A-1}<2|A| C=C_{1}, \quad \text { (say) }
$$

for any $\delta$ in between $\delta_{n}^{g}(x)$ and $\delta_{g}^{g}(x)$ (uniformly in $x$ ). So, by using the oneterm Taylor series expansion,

$$
\begin{aligned}
& \left|\sum_{x} K\left(\delta_{n}^{g}(x)\right) f_{\theta^{g}}^{1+\beta}(x) u_{j \theta^{g}}(x)-\sum_{x} K\left(\delta_{g}^{g}(x)\right) f_{\theta^{g}}^{1+\beta}(x) u_{j \theta^{g}}(x)\right| \\
\leq & C_{1} \sum_{x}\left|\delta_{n}^{g}(x)-\delta_{g}^{g}(x)\right| f_{\theta^{g}}^{1+\beta}(x)\left|u_{j \theta^{g}}(x)\right| .
\end{aligned}
$$

However, by Lemma 1(1),

$$
E\left[\left|\delta_{n}^{g}(x)-\delta_{g}^{g}(x)\right|\right] \leq \frac{\left(g(x)(1-g(x))^{1 / 2}\right.}{f_{\theta^{g}}(x) \sqrt{n}} \rightarrow 0 \quad \text { as } n \rightarrow \infty .
$$

and, by Lemma 1(2), we have

$$
\begin{aligned}
& E\left[C_{1} \sum_{x}\left|\delta_{n}^{g}(x)-\delta_{g}^{g}(x)\right| f_{\theta^{g}}^{1+\beta}(x)\left|u_{j \theta g}(x)\right|\right] \\
\leq & 2 C_{1} \sum_{x} g^{1 / 2}(x) f_{\theta^{g}}^{\beta}(x)\left|u_{j \theta^{g}}(x)\right|<\infty \quad \text { (by Assumption 5). }
\end{aligned}
$$

Hence, by DCT, we get,

$\left.E\left[\mid \sum_{x} K\left(\delta_{n}^{g}(x)\right) f_{\theta^{g}}^{1+\beta}(x) u_{j \theta^{g}}(x)-\sum_{x} K\left(\delta_{g}^{g}(x)\right) f_{\theta^{g}}^{1+\beta}(x) u_{j \theta^{g}}(x)\right]\right] \rightarrow 0 \quad$ as $n \rightarrow \infty$

so that by Markov inequality we have the desired claim.

By similar argument we can show

$$
\begin{array}{r}
\sum_{x} K\left(\delta_{n}^{g}(x)\right) f_{\theta^{g}}^{1+\beta}(x) \\
\stackrel{P}{\rightarrow} \sum_{x} K\left(\delta_{g}^{g}(x)\right) f_{\theta^{g}}^{1+\beta}(x) .
\end{array}
$$


Therefore, we have

$$
\left.\nabla_{j} \operatorname{LSD}\left(r_{n}, f_{\theta}\right)\right|_{\theta=\theta^{g}} \stackrel{P}{\rightarrow} 0 .
$$

Thus, with probability tending to one, $\left|S_{1}\right|<p a^{3}$, where $p$ is the dimension of $\theta$ and $a$ is the radius of $Q_{a}$.

Next we consider the quadratic term $S_{2}$. We have,

$$
\begin{aligned}
\frac{A}{1+\beta} \nabla_{j k} \operatorname{LSD}\left(r_{n}, f_{\theta}\right)= & (1+\beta)\left(\sum_{x} f_{\theta}^{1+\beta} u_{j \theta} u_{k \theta}\right)\left(\sum_{x} f_{\theta}^{1+\beta}\right)^{-1} \\
& -(1+\beta)\left(\sum_{x} f_{\theta}^{1+\beta} u_{j \theta}\right)\left(\sum_{x} f_{\theta}^{1+\beta} u_{k \theta}\right)\left(\sum_{x} f_{\theta}^{1+\beta}\right)^{-2} \\
& -B\left(\sum_{x} r_{n}^{A} f_{\theta}^{B} u_{j \theta} u_{k \theta}\right)\left(\sum_{x} r_{n}^{A} f_{\theta}^{B}\right)^{-1} \\
& +B\left(\sum_{x} r_{n}^{A} f_{\theta}^{B} u_{j \theta}\right)\left(\sum_{x} r_{n}^{A} f_{\theta}^{B} u_{k \theta}\right)\left(\sum_{x} r_{n}^{A} f_{\theta}^{B}\right)^{-2}(33)
\end{aligned}
$$

We will now show that

$$
\left.\nabla_{j k} \operatorname{LSD}\left(r_{n}, f_{\theta}\right)\right|_{\theta=\theta^{g}} \stackrel{P}{\rightarrow}-J_{g}^{j, k} .
$$

To show this we will show that

$$
\begin{aligned}
& \sum_{x} K\left(\delta_{n}^{g}(x)\right) f_{\theta^{g}}^{1+\beta}(x) u_{j \theta^{g}}(x) u_{k \theta^{g}}(x) \\
\rightarrow & \sum_{x} K\left(\delta_{g}^{g}(x)\right) f_{\theta^{g}}^{1+\beta}(x) u_{j \theta^{g}}(x) u_{k \theta^{g}}(x) .
\end{aligned}
$$

Note that by assumption 7 and using the fact that $r_{n}(x) \rightarrow g(x)$ a.s. by SLLN and by (28) we get the following expansion,

$$
\begin{aligned}
& \left|\sum_{x} K\left(\delta_{n}^{g}(x)\right) f_{\theta g}^{1+\beta}(x) u_{j \theta^{g}}(x) u_{k \theta^{g}}(x)-\sum_{x} K\left(\delta_{g}^{g}(x)\right) f_{\theta^{g}}^{1+\beta}(x) u_{j \theta^{g}}(x) u_{k \theta^{g}}(x)\right| \\
\leq & C_{1} \sum_{x}\left|\delta_{n}^{g}(x)-\delta_{g}^{g}(x)\right| f_{\theta^{g}}^{1+\beta}(x)\left|u_{j \theta^{g}}(x) u_{k \theta^{g}}(x)\right| .
\end{aligned}
$$

However, by Lemma 1(1),

$$
E\left[\left|\delta_{n}^{g}(x)-\delta_{g}^{g}(x)\right|\right] \leq \frac{\left(g(x)(1-g(x))^{1 / 2}\right.}{f_{\theta^{g}}(x) \sqrt{n}} \rightarrow 0 \quad \text { as } n \rightarrow \infty .
$$

and, by Lemma $1(2)$, we have

$$
\begin{aligned}
& E\left[C_{1} \sum_{x}\left|\delta_{n}^{g}(x)-\delta_{g}^{g}(x)\right| f_{\theta^{g}}^{1+\beta}(x)\left|u_{j \theta^{g}}(x) u_{k \theta^{g}}(x)\right|\right] \\
\leq & 2 C_{1} \sum_{x} g^{1 / 2}(x) f_{\theta^{g}}^{\beta}(x)\left|u_{j \theta^{g}}(x) u_{k \theta^{g}}(x)\right|<\infty \quad \text { (by Assumption 5). }
\end{aligned}
$$


Hence, by DCT, we get,

$$
\begin{aligned}
& \mid \sum_{x} K\left(\delta_{n}^{g}(x)\right) f_{\theta^{g}}^{1+\beta}(x) u_{j \theta^{g}}(x) u_{k \theta^{g}}(x) \\
- & \sum_{x} K\left(\delta_{g}^{g}(x)\right) f_{\theta^{g}}^{1+\beta}(x) u_{j \theta^{g}}(x) u_{k \theta^{g}}(x) \mid \rightarrow 0
\end{aligned}
$$

as $n \rightarrow \infty$. so that by Markov inequality we have the desired claim. and along with we will use

$$
\sum_{x} K\left(\delta_{n}^{g}(x)\right) f_{\theta^{g}}^{1+\beta}(x) u_{j \theta^{g}}(x) \stackrel{P}{\rightarrow} \sum_{x} K\left(\delta_{g}^{g}(x)\right) f_{\theta^{g}}^{1+\beta}(x) u_{j \theta^{g}}(x)
$$

and

$$
\sum_{x} K\left(\delta_{n}^{g}(x)\right) f_{\theta^{g}}^{1+\beta}(x) \stackrel{P}{\rightarrow} \sum_{x} K\left(\delta_{g}^{g}(x)\right) f_{\theta^{g}}^{1+\beta}(x)
$$

as $n \rightarrow \infty$ from the previous part of the proof. Thus, combining (37), (38) and (39), we get that,

$$
\left.\nabla_{j k} \operatorname{LSD}\left(r_{n}, f_{\theta}\right)\right|_{\theta=\theta^{g}} \stackrel{P}{\rightarrow}-J_{g}^{j, k} .
$$

Therefore,

$$
\begin{gathered}
2 S_{2}=\frac{1+\beta}{A} \sum_{j, k}\left\{\left.\nabla_{j k} \operatorname{LSD}\left(r_{n}, f_{\theta}\right)\right|_{\theta=\theta^{g}}-\left(-J_{g}^{j, k}\right)\right\}\left(\theta_{j}-\theta_{j}^{g}\right)\left(\theta_{k}-\theta_{k}^{g}\right) \\
+\sum_{j, k}\left\{-\left(\frac{(1+\beta)}{A} J_{g}^{j, k}\right)\left(\theta_{j}-\theta_{j}^{g}\right)\left(\theta_{k}-\theta_{k}^{g}\right)\right\} .
\end{gathered}
$$

Now the absolute value of the first term in above (41) is $<p^{2} a^{3}$ with probability tending to one. And, the second term in (41) is a negative definite quadratic form in the variables $\left(\theta_{j}-\theta_{j}^{g}\right)$. Letting $\gamma_{1}$ be the largest eigenvalue of $\frac{(1+\beta)}{A} J_{g}$, the quadratic form is $<\gamma_{1} a^{2}$. Combining the two terms, we see that there exists $c>0$ and $a_{0}>0$ such that for $a<a_{0}$, we have $S_{2}<-c a^{2}$ with 
probability tending to one. Finally, considering the cubic term $S_{3}$, we have

$$
\begin{aligned}
& \frac{A}{1+\beta} \nabla_{j k l} \operatorname{LSD}\left(r_{n}, f_{\theta}\right)=(1+\beta)^{2}\left(\sum_{x} f_{\theta}^{1+\beta} u_{j \theta} u_{k \theta} u_{l \theta}\right)\left(\sum_{x} f_{\theta}^{1+\beta}\right)^{-1} \\
& +(1+\beta)\left(\sum_{x} f_{\theta}^{1+\beta} u_{j l \theta} u_{k \theta}\right)\left(\sum_{x} f_{\theta}^{1+\beta}\right)^{-1}+(1+\beta)\left(\sum_{x} f_{\theta}^{1+\beta} u_{j \theta} u_{k l \theta}\right)\left(\sum_{x} f_{\theta}^{1+\beta}\right)^{-1} \\
& -(1+\beta)^{2}\left(\sum_{x} f_{\theta}^{1+\beta} u_{j \theta} u_{k \theta}\right)\left(\sum_{x} f_{\theta}^{1+\beta} u_{l \theta}\right)\left(\sum_{x} f_{\theta}^{1+\beta}\right)^{-2}+(1+\beta)\left(\sum_{x} f_{\theta}^{1+\beta} u_{l \theta} u_{j k \theta}\right)\left(\sum_{x} f_{\theta}^{1+\beta}\right)^{-1} \\
& +\left(\sum_{x} f_{\theta}^{1+\beta} u_{j k l \theta}\right)\left(\sum_{x} f_{\theta}^{1+\beta}\right)^{-1}-(1+\beta)\left(\sum_{x} f_{\theta}^{1+\beta} u_{j k \theta}\right)\left(\sum_{x} f_{\theta}^{1+\beta} u_{l \theta}\right)\left(\sum_{x} f_{\theta}^{1+\beta}\right)^{-2} \\
& -(1+\beta)^{2}\left(\sum_{x} f_{\theta}^{1+\beta} u_{j \theta} u_{l \theta}\right)\left(\sum_{x} f_{\theta}^{1+\beta} u_{k \theta}\right)\left(\sum_{x} f_{\theta}^{1+\beta}\right)^{-2} \\
& -(1+\beta)\left(\sum_{x} f_{\theta}^{1+\beta} u_{j l \theta}\right)\left(\sum_{x} f_{\theta}^{1+\beta} u_{k \theta}\right)\left(\sum_{x} f_{\theta}^{1+\beta}\right)^{-2} \\
& -(1+\beta)^{2}\left(\sum_{x} f_{\theta}^{1+\beta} u_{j \theta}\right)\left(\sum_{x} f_{\theta}^{1+\beta} u_{k \theta} u_{l \theta}\right)\left(\sum_{x} f_{\theta}^{1+\beta}\right)^{-2} \\
& -(1+\beta)\left(\sum_{x} f_{\theta}^{1+\beta} u_{j \theta}\right)\left(\sum_{x} f_{\theta}^{1+\beta} u_{k l \theta}\right)\left(\sum_{x} f_{\theta}^{1+\beta}\right)^{-2} \\
& +2(1+\beta)^{2}\left(\sum_{x} f_{\theta}^{1+\beta} u_{j \theta}\right)\left(\sum_{x} f_{\theta}^{1+\beta} u_{k \theta}\right)\left(\sum_{x} f_{\theta}^{1+\beta} u_{l \theta}\right)\left(\sum_{x} f_{\theta}^{1+\beta}\right)^{-3} \\
& -B^{2}\left(\sum_{x} r_{n}^{A} f_{\theta}^{B} u_{j \theta} u_{k \theta} u_{l \theta}\right)\left(\sum_{x} r_{n}^{A} f_{\theta}^{B}\right)^{-1}-B\left(\sum_{x} r_{n}^{A} f_{\theta}^{B} u_{j l \theta} u_{k \theta}\right)\left(\sum_{x} r_{n}^{A} f_{\theta}^{B}\right)^{-1} \\
& -B\left(\sum_{x} r_{n}^{A} f_{\theta}^{B} u_{j \theta} u_{k l \theta}\right)\left(\sum_{x} r_{n}^{A} f_{\theta}^{B}\right)^{-1}+B^{2}\left(\sum_{x} r_{n}^{A} f_{\theta}^{B} u_{j \theta} u_{k \theta}\right)\left(\sum_{x} r_{n}^{A} f_{\theta}^{B} u_{l \theta}\right)\left(\sum_{x} r_{n}^{A} f_{\theta}^{B}\right)^{-2} \\
& -B\left(\sum_{x} r_{n}^{A} f_{\theta}^{B} u_{j k \theta} u_{l \theta}\right)\left(\sum_{x} r_{n}^{A} f_{\theta}^{B} u_{j k l \theta}\right)\left(\sum_{x} r_{n}^{A} f_{\theta}^{B}\right)^{-2} \\
& +B\left(\sum_{x} r_{n}^{A} f_{\theta}^{B} u_{j k \theta}\right)\left(\sum_{x} r_{n}^{A} f_{\theta}^{B} u_{l \theta}\right)\left(\sum_{x} r_{n}^{A} f_{\theta}^{B}\right)^{-2} \\
& +B^{2}\left(\sum_{x} r_{n}^{A} f_{\theta}^{B} u_{j \theta} u_{l \theta}\right)\left(\sum_{x} r_{n}^{A} f_{\theta}^{B} u_{k \theta}\right)\left(\sum_{x} r_{n}^{A} f_{\theta}^{B}\right)^{-2} \\
& +B\left(\sum_{x} r_{n}^{A} f_{\theta}^{B} u_{j l \theta}\right)\left(\sum_{x} r_{n}^{A} f_{\theta}^{B} u_{k \theta}\right)\left(\sum_{x} r_{n}^{A} f_{\theta}^{B}\right)^{-2} \\
& +B^{2}\left(\sum_{x} r_{n}^{A} f_{\theta}^{B} u_{k \theta} u_{l \theta}\right)\left(\sum_{x} r_{n}^{A} f_{\theta}^{B} u_{j \theta}\right)\left(\sum_{x} r_{n}^{A} f_{\theta}^{B}\right)^{-2}
\end{aligned}
$$




$$
\begin{array}{r}
+B\left(\sum_{x} r_{n}^{A} f_{\theta}^{B} u_{k l \theta}\right)\left(\sum_{x} r_{n}^{A} f_{\theta}^{B} u_{j \theta}\right)\left(\sum_{x} r_{n}^{A} f_{\theta}^{B}\right)^{-2} \\
-2 B^{2}\left(\sum_{x} r_{n}^{A} f_{\theta}^{B} u_{j \theta}\right)\left(\sum_{x} r_{n}^{A} f_{\theta}^{B} u_{k \theta}\right)\left(\sum_{x} r_{n}^{A} f_{\theta}^{B} u_{l \theta}\right)\left(\sum_{x} r_{n}^{A} f_{\theta}^{B}\right)^{-3} .
\end{array}
$$

Using the assumptions and $r_{n}(x) \rightarrow g(x)$ we can show the cubic term $S_{3}$ is also bounded. Hence, we have $\left|S_{3}\right|<b a^{3}$ on the sphere $Q_{a}$ with probability tending to one. Combining the three inequality we get that

$$
\max \left(S_{1}+S_{2}+S_{3}\right)<-c a^{2}+(b+p) a^{3} \quad\left[<0 \quad \text { for } \quad a<\frac{c}{b+p}\right] .
$$

Thus, for any sufficiently small $a$, there exists a sequence of roots $\theta_{n}=\theta_{n}(a)$ to the minimum LSD estimating equation such that $P\left(\left\|\theta_{n}-\theta^{g}\right\|_{2}<a\right)$ converges to one, where $\|.\|_{2}$ denotes the $L_{2}$-norm. It remains to show that we can determine such a sequence independent of $a$. For let $\theta_{n}^{*}$ be the root which is closest to $\theta^{g}$. This exists because the limit of a sequence of roots is again a root by the continuity of the LSD. Hence proved the consistency part. 